PEFERENOE

Applied and

NISTIR 4679

Computational

Mathematics

Division

The Effect of Gravity Modulation on Thermosolutal Convection in an Infinite Layer of Fluid

B.V. Saunders, B.T. Murray,

G.B. McFadden, S.R. Coriell, and A.A. Wheeler

October 1991

$-Q C$ 100

.456

\#4679

1991

U.S. DEPARTMENT OF COMMERCE National Institute of Standards and Technology Gaithersburg, MD 20899 



\section{The Effect of Gravity Modulation on Thermosolutal Convection in an Infinite Layer of Fluid}

\author{
B. V. Saunders \\ B. T. Murray \\ G. B. McFadden \\ S. R. Coriell \\ A. A. Wheeler
}
U.S. DEPARTMENT OF COMMERCE National Institute of Standards and Technology Computing and Applied Mathematics Laboratory
Applied and Computational Mathematics Division
Gaithersburg, MD 20899

October 1991

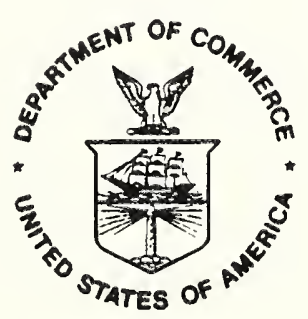

U.S. DEPARTMENT OF COMMERCE Robert A. Mosbacher, Secretary NATIONAL INSTITUTE OF STANDARDS AND TECHNOLOGY

John W. Lyons, Director 



\title{
The Effect of Gravity Modulation on Thermosolutal Convection in an Infinite Layer of Fluid
}

\author{
B. V. Saunders, B. T. Murray, \\ G. B. McFadden, S. R. Coriell, and A. A. Wheeler \\ National Institute of Standards and Technology \\ Gaithersburg, Maryland 20899, USA
}

\begin{abstract}
The effect of time-periodic vertical gravity modulation on the onset of thermosolutal convection in an infinite horizontal layer with stress-free boundaries is investigated using Floquet theory for the linear stability analysis. We consider situations for which the fluid layer is stably stratified in either the fingering or diffusive regimes of double-diffusive convection. Results are presented both with and without steady background acceleration. Modulation may stabilize an unstable base solution or destabilize a stable base solution. In addition to synchronous and subharmonic response to the modulation frequency, instability in the double-diffusive system can occur via a complex conjugate mode. In the diffusive regime, where oscillatory onset occurs in the unmodulated system, regions of resonant instability occur and exhibit strong coupling with the unmodulated oscillatory frequency. The response to modulation of the fundamental instability of the unmodulated system is described both analytically and numerically; in the double-diffusive system this mode persists under subcritical conditions as a high-frequency lobe.
\end{abstract}




\section{Introduction}

When a fluid in a gravitational field contains two components of different diffusivities that affect the density, for example, temperature and solute, a broader range of dynamical behavior is found in the convective instabilities that may occur. This phenomenon, known variously as thermosolutal convection, double-diffusive convection, or thermohaline convection when the solute is salt, has been studied extensively since it was first described by Stommel et al. in 1956 [1]. The early work on the problem is summarized in several reviews $[2,3,4]$. Although initial research in this area was in the field of oceanography, the study of double-diffusive convection is of practical importance in many diverse areas involving convective heat and mass transfer, including astrophysics, geophysics, geology, chemistry, and metallurgy [5].

The current work is motivated by the desire to better understand the conditions for which convective instabilities can occur in particular configurations used for solidification of binary alloys. The onset of thermosolutal convection is relevant to the process of directional solidification via the vertical Bridgman technique, where the inherent temperature and solute gradients in the melt are aligned with the gravity vector. Buoyant convection resulting from thermosolutal instabilities is undesirable, since the convection alters the distribution of solute which can lead to macrosegregation in the solid. Coriell et al. [6] examined the onset of thermosolutal convection in the liquid and morphological instability of the solidliquid interface during the vertical directional solidification of a binary alloy in a constant gravitational field. The model accounts for the fact that the solid-liquid interface moves and has an unconstrained shape. In this configuration, the unperturbed temperature and solute profiles vary exponentially with the distance from the interface as opposed to the linear variation most often considered in treatments of thermosolutal convection. The onset of convective and morphological instability in models of vertical directional solidification has been the subject of numerous studies $[6,7,8,9,10,11,12]$. A review article by Davis contains a more complete list of references [13].

It has been found that for many alloys, even when interfacial instabilities can be ignored, it is difficult to avoid convection in the earth's gravitational field. Consequently, this has led to research into the possibility of processing materials in space where the low level background gravitational acceleration $\left(10^{-6}\right.$ of the earth's gravitational acceleration, $\left.g_{e}\right)$ can eliminate buoyancy driven convection. However, research has shown $[14,15,16]$ that timedependent accelerations ( $\mathrm{g}$-jitter) of substantial amplitude resulting from orbital maneuvers and inherent mechanical vibrations may alone induce buoyant convection. It is also of interest to understand how vibration might be used to control convective instabilities. Recently, low frequency vibrations have been used in attempts to control the shape and position of the solid-liquid interface during Bridgman growth $[17,18]$. A brief literature review of the use of vibrations in crystal growth is given by Zharikov et al. [19]

The effect of time-dependent gravitational acceleration on the onset of convection in the Rayleigh-Benard configuration has been the subject of several investigations by Gershuni and Zhukhovitskii with various co-workers. Their book [20] provides a comprehensive introduction to the study of the effects of time-dependent modulation, including the 
method of averaging for analysis of the high frequency limit and numerical computations of the nonlinear behavior. Gresho and Sani [21] also determined the onset conditions for the Rayleigh-Benard problem. More recent investigations of the nonlinear behavior have also been performed [22, 23]. There are several references on related problems involving time-dependent modulation $[24,25,26,27,28,29]$.

Murray et al. [30] considered the effect of sinusoidal time-dependent accelerations on the onset of solutal convection during directional solidification of a binary alloy at constant velocity. The processing conditions were assumed to be such that the thermal Rayleigh number was sufficiently small so that thermal effects could be ignored. The study showed that a stable base state can be made unstable by the modulation, while an unstable base state can be made stable. Ideally, a study of the effect of temporal modulation on thermosolutal convection in a model similar to the one used by Coriell et al. [6] is desired; however, it would require extensive computations and the number of parameters would preclude a systematic study. Thus, in the present paper, in order to gain better understanding of this complex problem we examine the effect of sinusoidal time-dependent accelerations on the onset of thermosolutal convection in an infinite horizontal layer of fluid. In particular, we consider the case of stress-free boundary conditions at the top and bottom of the layer, for which there are exact spatial eigenfunctions so that the linear stability problem reduces to solving a system of ordinary differential equations in time with periodic coefficients. This paper can be viewed as a first step in expanding the previous study [30] to the full problem of examining the effects of gravity modulation on thermosolutal convection during the directional solidification of binary alloys.

Although the stability analysis for this idealized configuration presents no computational difficulties, it is not easy to predict and interpret the results for this problem, since it involves the combined effect of gravity modulation on both the thermal and solutal fields. When opposing temperature and solute gradients are present in a constant gravitational field, the difference in diffusivities causes two distinct regimes to occur depending on which gradient is stabilizing. In the fingering regime, which occurs when the component with the smaller diffusivity is destabilizing (solute), the onset of convection is characterized by a monotonic increase in the size of the disturbances. When temperature is destabilizing, the diffusive or overstable regime appears and instability occurs via a Hopf bifurcation with disturbances growing in an oscillatory fashion. Although the fingering regime is the more common situation for the directional solidification problem, it is of interest to study how modulation affects the oscillatory onset of the diffusive regime.

Despite the fact that the computations are relatively easy to perform, there are enough parameters to explore that it is difficult to obtain a definitive understanding of the problem. Instead, we present the results from many numerical calculations using a small but reasonable set of values for these parameters, and interpret those features that emerge from the calculations. For example, the fact that for the double-diffusive system there are three Floquet multipliers, rather than just two as in the single component case, allows the overall topology of the marginal stability curves to be more complicated; the single component case is described by the damped Mathieu equation [20]. 
We find that in the fingering regime, the stability results are generally reminiscent of those for the damped Mathieu equation, in that both synchronous and subharmonic "resonant modes" are found for the modulated system. However, the detailed behavior of the modulated system under conditions for which the unmodulated system is marginally stable are somewhat different, and the response to modulation of the "fundamental mode" associated with these near-critical conditions is more complicated in the double-diffusive system. In particular, we find that the fundamental mode can still persist under sub-critical conditions of the unmodulated system; its behavior for near-critical conditions can be described analytically for small modulation amplitudes.

Another question we explore is the behavior under modulation of a system which in the absence of modulation undergoes instability by a time-dependent Hopf bifurcation; in this case a resonant behavior might be anticipated when the forcing frequency and the natural frequency of the unmodulated system are related. Indeed, in the diffusive regime, we find that there can be a significant destabilization of the "resonant modes" of the modulated system under near-critical conditions of the unmodulated state, precisely at multiples of the natural frequency of the unmodulated system. Here the usual terminology unfortunately is somewhat confusing: traditionally the parametric instabilities of the modulated system that are observed at sufficiently large modulation amplitudes under conditions for which the unmodulated system is stable are referred to as "resonant modes," and these modes are also observed in the diffusive regime of double-diffusive convection. Here we are examining conditions under which these modes are destabilized due to a significant resonant reaction to the natural frequencies of the unmodulated system. There is also a complicated response of the fundamental mode itself under these conditions, which is analogous to that observed in the fingering case. Again, these phenomena can be described analytically for small modulation amplitudes.

In Section 2 we describe the governing equations for the model and outline the linear stability analysis. The periodic coefficients in the linear stability equations permit the use of Floquet theory to compute the onset conditions for instability. In Section 3 we present some characteristic results of the calculations and in Section 4 summarize the results. Some details of associated expansion procedures are summarized in Appendices I and II.

\section{Governing equations}

The present paper considers the effect of a sinusoidal temporal modulation of the vertical gravitational field on the onset of thermosolutal convection in an infinite horizontal layer of fluid. For mathematical convenience both the top and bottom boundaries are assumed to be stress-free, with the temperature and solute concentration there fixed. Assuming an incompressible Newtonian fluid, the governing equations are the conservation of mass equation, the Navier-Stokes equations, the convection-diffusion equation for the solute concentration $c$, the convection-diffusion equation for the temperature field $\mathrm{T}$, and a linear equation of state expressing the dependence of the melt density $\rho$ on solute concentration and temperature variation. Furthermore, the Boussinesq approximation is employed so that variations 
in density are ignored except in the body force term in the Navier-Stokes equations. The equations are

$$
\begin{gathered}
\nabla \cdot \boldsymbol{u}=0 \\
\frac{\partial \boldsymbol{u}}{\partial t}+(\boldsymbol{u} \cdot \nabla) \boldsymbol{u}=-\nabla p / \rho_{0}+\nu \nabla^{2} \boldsymbol{u}+\boldsymbol{g} \rho / \rho_{0} \\
\frac{\partial c}{\partial t}+(\boldsymbol{u} \cdot \nabla) c=D \nabla^{2} c \\
\frac{\partial T}{\partial t}+(\boldsymbol{u} \cdot \nabla) T=\kappa \nabla^{2} T \\
\rho=\rho_{0}\left[1-\alpha\left(T-T_{0}\right)+\beta\left(c-c_{0}\right)\right]
\end{gathered}
$$

where $\mathrm{p}$ is the fluid pressure, $\rho_{0}$ is the density at the lower surface, $\nu$ is the kinematic viscosity, $\mathrm{D}$ is the solute diffusion coefficient, $\kappa$ is the thermal diffusion coefficient, $c_{0}$ and $T_{0}$ are the solute concentration and temperature at the lower surface, and $\alpha$ and $\beta$ are volume expansion coefficients due to temperature and solute concentration variations, respectively. The gravitational field is taken to be

$$
\boldsymbol{g}=-g(\delta+\epsilon \cos \Omega t) \hat{\boldsymbol{z}},
$$

where $g$ is a reference acceleration level, $\delta$ and $\epsilon$ are the relative amplitudes of the steady and modulated components of the acceleration, respectively, $\Omega$ is the modulation frequency, and $\hat{\boldsymbol{z}}$ is the unit vector in the vertical direction. Here, the modulation amplitude $\epsilon$ varies continuously, while $\delta$ takes on the values 0 or 1 only; the value $\delta=0$ represents no steady background acceleration level, while $\delta=1$ corresponds to terrestrial conditions.

The steady-state base solution consists of no fluid motion, with the temperature and solute fields given by the expressions

$$
\begin{aligned}
T^{(0)}(z) & =T_{0}+(z / d) \Delta T, \\
c^{(0)}(z) & =c_{0}+(z / d) \Delta c,
\end{aligned}
$$

where $d$ is the thickness of the layer, and $\Delta T=T_{1}-T_{0}$ and $\Delta c=c_{1}-c_{0}$ are the differences in temperature and solute across the layer with $T_{1}$ and $c_{1}$ being the temperature and solute concentration at the top surface. The pressure $p^{(0)}(z)$ in the undisturbed fluid is hydrostatic.

Dimensionless variables are defined in terms of the length scale $d$, the time scale $d^{2} / \nu$, the velocity scale $\nu / d$, the temperature scale $\Delta T$, and the solute scale $\Delta c$; from henceforth, all variables will be dimensionless. For the linear stability analysis of the base state we write the flow field variables as the sum of the base state and a perturbation quantity that is Fourier analyzed in the lateral directions. We have

$$
\left(\begin{array}{c}
u(x, y, z, t) \\
v(x, y, z, t) \\
w(x, y, z, t) \\
p(x, y, z, t) \\
T(x, y, z, t) \\
c(x, y, z, t)
\end{array}\right)=\left(\begin{array}{c}
0 \\
0 \\
0 \\
p^{(0)}(z) \\
T^{(0)}(z) \\
c^{(0)}(z)
\end{array}\right)+\left(\begin{array}{c}
u^{(1)}(z, t) \\
v^{(1)}(z, t) \\
w^{(1)}(z, t) \\
p^{(1)}(z, t) \\
T^{(1)}(z, t) \\
c^{(1)}(z, t)
\end{array}\right) \exp \left(i a_{x} x+i a_{y} y\right)
$$


where $\left(a_{x}, a_{y}\right)$ is the dimensionless horizontal wavenumber and $(u, v, w)$ are the components of the velocity field in the $(x, y, z)$ frame.

Substituting these quantities into the governing equations and linearizing yields

$$
\begin{gathered}
i a_{x} u^{(1)}+i a_{y} v^{(1)}+L w^{(1)}=0, \\
\frac{\partial u^{(1)}}{\partial t}+i a_{x} p^{(1)}=\left(L^{2}-a^{2}\right) u^{(1)}, \\
\frac{\partial v^{(1)}}{\partial t}+i a_{y} p^{(1)}=\left(L^{2}-a^{2}\right) v^{(1)}, \\
\frac{\partial w^{(1)}}{\partial t}+L p^{(1)}=\left(L^{2}-a^{2}\right) w^{(1)}+\operatorname{Ra}(\delta+\epsilon \cos \omega t) \frac{T^{(1)}}{\operatorname{Pr}}-\operatorname{Rs}(\delta+\epsilon \cos \omega t) \frac{c^{(1)}}{\mathrm{Sc}}, \\
\frac{\partial T^{(1)}}{\partial t}+w^{(1)}=\left(L^{2}-a^{2}\right) \frac{T^{(1)}}{\operatorname{Pr}}, \\
\frac{\partial c^{(1)}}{\partial t}+w^{(1)}=\left(L^{2}-a^{2}\right) \frac{c^{(1)}}{\operatorname{Sc}},
\end{gathered}
$$

where $L$ refers to the spatial derivative $(\partial / \partial z)$. The thermal and solutal Rayleigh numbers are

$$
\begin{aligned}
& \mathrm{Ra}=\frac{g \alpha \Delta T d^{3}}{\nu \kappa}, \\
& \mathrm{Rs}=\frac{g \beta \Delta c d^{3}}{\nu D},
\end{aligned}
$$

where with the chosen sign conventions, positive Rs and negative Ra correspond to unstable density stratification when $\boldsymbol{g}$ is downward. The remaining dimensionless parameters are the driving frequency $\omega=\Omega d^{2} / \nu$, the modulus of the horizontal wavenumber $a=\sqrt{a_{x}^{2}+a_{y}^{2}}$, the Prandtl number $\operatorname{Pr}=\nu / \kappa$, and the Schmidt number $\mathrm{Sc}=\nu / D$. A list of the dimensional and dimensionless parameters are given in Table I.

Through the use of the curl operator and algebraic manipulations [31], one can eliminate the horizontal velocity components and the pressure from (5) to reduce the system to

$$
\left(L^{2}-a^{2}\right) \frac{\partial w^{(1)}}{\partial t}=\left(L^{2}-a^{2}\right)^{2} w^{(1)}-a^{2} \operatorname{Ra}(\delta+\epsilon \cos \omega t) \frac{T^{(1)}}{\operatorname{Pr}}+a^{2} \operatorname{Rs}(\delta+\epsilon \cos \omega t) \frac{c^{(1)}}{\mathrm{Sc}}
$$

plus the second-order equations for the perturbed temperature, $T^{(1)}$, and the perturbed solute concentration, $c^{(1)}$.

Since we assume the tangential stresses vanish along the upper and lower surfaces, the vertical component of the flow satisfies $w^{(1)}=L^{2} w^{(1)}=0$ with $T^{(1)}=c^{(1)}=0$ at $z=0$ and $z=1$. These conditions are satisfied if the vertical dependence of the perturbations is assumed to have the form $\sin n \pi z$. This results in the ordinary differential equations

$$
\left(n^{2} \pi^{2}+a^{2}\right) \frac{d W}{d t}=-\left(n^{2} \pi^{2}+a^{2}\right)^{2} W+a^{2} \operatorname{Ra}(\delta+\epsilon \cos \omega t) \frac{\Theta}{\operatorname{Pr}}-a^{2} \operatorname{Rs}(\delta+\epsilon \cos \omega t) \frac{C}{\mathrm{Sc}},
$$




$$
\begin{aligned}
& \frac{d \Theta}{d t}=-\left(n^{2} \pi^{2}+a^{2}\right) \frac{\Theta}{\operatorname{Pr}}-W, \\
& \frac{d C}{d t}=-\left(n^{2} \pi^{2}+a^{2}\right) \frac{C}{\mathrm{Sc}}-W,
\end{aligned}
$$

where we have written $w^{(1)}(z, t)=W(t) \sin n \pi z, T^{(1)}(z, t)=\Theta(t) \sin n \pi z$, and $c^{(1)}(z, t)=$ $C(t) \sin n \pi z$.

Since the coefficients in (6) are either constant or periodic in $t$, using Floquet theory [32] one can obtain solutions of the form

$$
\left(\begin{array}{c}
W(t) \\
\Theta(t) \\
C(t)
\end{array}\right)=e^{\sigma t}\left(\begin{array}{c}
\hat{W}(t) \\
\hat{\Theta}(t) \\
\hat{C}(t)
\end{array}\right)
$$

where $\hat{W}, \hat{\Theta}$, and $\hat{C}$ are periodic in $t$ with period $2 \pi / \omega$ and $\sigma=\sigma_{r}+i \sigma_{i}$ is the complex growth rate. To calculate $\sigma$, we use the computer code DDRIV2 [33], which uses high-order Adams methods or Gear methods (for stiff problems) to solve a system of first-order differential equations. We choose convenient initial conditions and compute three linearly independent solutions for the unknowns at the end of one forcing period. These fundamental solutions form the columns of a $3 \times 3$ matrix, the eigenvalues of which are the Floquet multipliers, $e^{\sigma 2 \pi / \omega}$, from which $\sigma$ can be computed. The base state is linearly stable for a given set of parameters if, for all three eigenmodes, the real part of $\sigma$ is negative for all disturbances. To study the effect of modulation on the stability of the base state, neutral curves are obtained by varying one of the parameters (e.g., modulation amplitude $\epsilon$ ) with the remaining parameters fixed to determine where $\sigma_{r}=0$.

\section{Results}

Before presenting results on the effect of modulation on the onset of thermosolutal convection, it is helpful to review the essential results for the unmodulated problem $(\epsilon=0)$. In this case, a cubic dispersion relation in terms of the complex growth rate $\sigma$ is obtained [2]. Analysis of this relation yields the stability criteria in terms of $\mathrm{Ra}$ and $\mathrm{Rs}$ for given values of $a, \mathrm{Pr}$, and Sc.

For our definition of the parameters, when $\mathrm{Ra}$ and $\mathrm{Rs}$ are positive, the solute field is destabilizing while the thermal field is stabilizing. This is the fingering regime which is characterized by steady onset. The critical value Rs* of the solutal Rayleigh number for steady onset is given by the linear relation

$$
R s^{*}=\frac{27 \pi^{4}}{4}+R a,
$$

which is independent of the Prandtl and Schmidt numbers. When $\mathrm{Ra}=0$ equation (8) reduces to the critical Rayleigh number for the single component case $\left(R_{s}^{*}=27 \pi^{4} / 4\right)$ with stress-free boundaries [31]. 
In the diffusive regime, $\mathrm{Ra}$ and $\mathrm{Rs}$ are negative and the thermal field is destabilizing. The onset of time-dependent oscillatory instability occurs when

$$
R s^{*}=\frac{27 \pi^{4}}{4} \frac{(\mathrm{Pr}+\mathrm{Sc})(\mathrm{Pr}+1)}{\mathrm{Pr}^{2}}+\frac{\mathrm{Sc}^{2}}{\mathrm{Pr}^{2}} \frac{\mathrm{Pr}+1}{\mathrm{Sc}+1} \mathrm{Ra}
$$

and the frequency of oscillation is given by

$$
\sigma_{i}^{2}=\frac{1}{3}\left[\frac{27 \pi^{4}}{4}\left(\frac{1}{\mathrm{Pr}}+\frac{1}{\mathrm{Sc}}+\frac{1}{\mathrm{PrSc}}\right)+\frac{\mathrm{Ra}}{\mathrm{Pr}}-\frac{\mathrm{Rs}}{\mathrm{Sc}}\right] .
$$

In both regimes, the critical wavenumber is given by $a=\pi / \sqrt{2}$. Fig. 1 shows the stability boundaries in the $(\mathrm{Ra}, \mathrm{Rs})$ plane in the absence of modulation. The solid curve is the steady onset curve (8); the dashed curve corresponds to oscillatory onset (9). The oscillatory onset curve is shown for $\operatorname{Pr}=1$ and $\mathrm{Sc}=10$. The dependence of the slope of the oscillatory onset curve on $\mathrm{Pr}$ and Sc typically yields large values of the slope for materials of practical interest, and so, in general, it is difficult to show the stability regions of interest for both onset modes on the same plot.

Another relevant frequency for a stably stratified system is the buoyancy or Brunt-Väisälä frequency [2] which in our notation is given by

$$
N=\sqrt{\mathrm{Ra} / \mathrm{Pr}-\mathrm{Rs} / \mathrm{Sc}} .
$$

This frequency is contained in the double-diffusive oscillation frequency, $\sigma_{i}$, and may also be important with regards to resonance phenomena resulting from the modulation.

We present results from the linear theory for the effect of sinusoidal modulation. In principle, the critical wavenumber for instability should be determined by scanning the entire range of wavenumbers. For the results presented here, the wavenumber is fixed at the unmodulated critical value $(n=1$ and $a=\pi / \sqrt{2})$ in order to explore more fully the dependence on the other parameters. Alternatively, one could adopt the viewpoint that the wavenumber has been fixed by imposing lateral boundary conditions that represent flow in an idealized box with stress-free sidewalls. For the first set of results, we consider the effect of sinusoidal modulation on the onset of convection in the single component (thermal problem) to serve as a reference for the thermosolutal calculations. Also, we begin by considering a case relevant to a low gravity environment, and assurne the steady component of gravity is negligible. We set $\delta=0$ and look for values of the relative modulation frequency, $\epsilon$, that lead to instability for given values of the modulation frequency, $\omega$. To obtain the results for thermal convection only, we set $R s=0$ in (6a). We note that with $\delta=0$, the results depend only on the product $\epsilon \mathrm{Ra}$ which represents the overall modulation amplitude.

In Fig. 2 we plot $\epsilon$ versus the inverse of the modulation frequency to show the neutral stability boundaries (where $\sigma_{r}=0$ ) for $\operatorname{Ra}=1000$ and $\operatorname{Pr}=0.1$. The shaded regions indicate where the sinusoidal modulation destabilizes an otherwise stable configuration. The linear stability problem for the single component case reduces to the damped Mathieu equation as 
discussed in detail in Gershuni and Zhukhovitskii [20]. The lobe-shaped shaded regions alternate between subharmonic $\left(\sigma_{i}=\omega / 2\right)$ and synchronous $\left(\sigma_{i}=0\right)$ resonant response which is characteristic of solutions to the Mathieu equation and similar Floquet systems. The regions are shaded with vertical lines to indicate a subharmonic resonant response or horizontal lines to indicate a synchronous resonant response. The minimum amplitude required for instability $(\epsilon \mathrm{Ra}=7290)$ occurs at a dimensionless frequency $\omega$ around 105 . This amplitude corresponds to approximately eleven times the unmodulated critical level of $27 \pi^{4} / 4$. The minimum amplitude for instability of the remaining lobes increases as the frequency decreases. A similar case was presented in Gershuni and Zhukhovitskii [20], although for a different value of the Prandtl number; the same qualitative behavior as shown in Fig. 2 was obtained. The value of the Prandtl number controls the level of damping, and hence the amplitude of modulation required for destabilization. At very large frequencies, the system is unable to react to the modulation and the state of the unmodulated system is approached. For resonant behavior at large frequencies, an analysis of the governing equations indicates that $\epsilon \mathrm{Ra} \sim \omega^{2}$ in the limit $\omega \rightarrow \infty[34]$.

\subsection{Fingering Regime}

For the thermosolutal problem we first consider parameters corresponding to the fingering regime in the unmodulated case where the solutal field is the destabilizing component. For $\mathrm{Ra}=1000$, the critical solutal Rayleigh number is 1657.5 . Again, we start with a case of no background acceleration $(\delta=0)$. Fig. 3 shows the neutral stability boundaries for the modulated solution when $\delta=0, \mathrm{Ra}=1000$, and $\mathrm{Rs}=1690$. The Prandtl and Schmidt numbers are fixed at 0.1 and 10 , respectively, for the modulation calculations. These values are a reasonable approximation for semiconductor-type materials. As before, the vertical and horizontal shading lines indicate that the onset of convection is subharmonic and synchronous, respectively. Intersecting lines indicate doubly unstable regions, that is, areas where two of the three growth rates $\sigma_{r}$ are positive. Diagonally intersecting lines indicate an oscillatory response where the onset of convection occurs as a complex conjugate pair, while intersecting vertical and horizontal lines indicate both a subharmonic and a synchronous instability. In Fig. 3 the lobe which exists for the highest frequencies shows a synchronous response to the modulation, but this region is overlapped by a doubly unstable area where a subharmonic response also appears. Adjacent to this region is a lobe where the onset of instability appears as a complex conjugate pair. The two doubly unstable regions in Fig. 3 are similar to the first two lobes seen in the single component thermal problem in Fig. 2. The last region for the lower frequencies shown in Fig. 3 shows a subharmonic response but it is no longer lobe-shaped.

We now let $\delta=1$, so that the steady component of gravity is significant. Then the problem is equivalent to looking at the effect of a sinusoidal modulation with a one-g background level. With no modulation, the critical solutal Rayleigh number is 1657.5 , so $R s=1690$ is slightly unstable for $\epsilon=0$. In Fig. 4 we show the neutral stability regions for this case ( $\delta$ $=1, \mathrm{Ra}=1000$, and $\mathrm{Rs}=1690)$. Because this case is unstable without modulation, there is what is referred to as a fundamental region of instability [20], which is synchronous for 
all frequencies, as indicated by the horizontal shading lines. It is possible to stabilize the fundamental mode of instability with an $\epsilon$ that depends on frequency, with larger frequencies requiring larger modulation amplitudes. However, the stabilization is bounded above by lobes or bands of resonant instability. The structure of the resonant bands is qualitatively similar to the regions found for the same Rayleigh numbers with $\delta=0$ in Fig. 3 . This indicates that the resonant behavior is somewhat insensitive to the level of the steady background acceleration.

In Fig. 5 we show the neutral stability curves obtained when modulation is applied for the same parameters as in Fig. 4, except that $\mathrm{Rs}$ has been reduced to the unmodulated subcritical value of 1624 . We find that the subharmonic and complex conjugate resonant lobes and the broad subharmonic region at lower frequencies are qualitatively. similar for the two different $\mathrm{Rs}$ values. The primary difference is the change in the synchronous fundamental region, which is now a lobe region at $R s=1624$. For Figs. $2-5$, the buoyancy frequency (11) is approximately 100 , which is in the vicinity of the highest frequency subharmonic resonant lobe.

For $\delta=1$, a transition in the stability of the modulated system for small values of $\epsilon$ is apparent in comparing Figs. 4 and 5. The transition occurs as the solutal Rayleigh number exceeds its unmodulated critical value $\mathrm{Rs}^{*}$. For $\mathrm{Rs}<\mathrm{Rs}^{*}$, the axis $\epsilon=0$ lies in the stable region, whereas for $R s>R^{*}$ this axis lies in the unstable region. The transitional behavior is shown in more detail in Fig. 6, where it is evident that the marginal stability curves for $\mathrm{Rs}<\mathrm{Rs}^{*}$ and $\mathrm{Rs}>\mathrm{Rs}^{*}$ are tending toward a common asymptote or separatrix as $\mathrm{Rs}$ tends to $\mathrm{Rs}^{*}$; this separatrix is shown as the dashed line in Fig. 6 with $1 / \omega \approx 0.013$ at $\epsilon=0$.

The specific value of $\omega=\omega_{0}$ for the separatrix at $\epsilon=0$ can be determined through an expansion procedure in $\epsilon$. For $R s=R s^{*}$, the separatrix describes a time-periodic solution to the linearized governing equations, which as $\epsilon \rightarrow 0$ tends to the marginally-stable steadystate eigenmode

$$
\left(\begin{array}{c}
W_{0} \\
\Theta_{0} \\
C_{0}
\end{array}\right)=\left(\begin{array}{c}
1 \\
-\operatorname{Pr} /\left(\pi^{2}+a^{2}\right) \\
-\operatorname{Sc} /\left(\pi^{2}+a^{2}\right)
\end{array}\right)
$$

of the unmodulated system. For fixed values of $R a$ and $R s=R s^{*}$, the solution is expanded formally in powers of $\epsilon$,

$$
\left(\begin{array}{c}
W(t, \epsilon) \\
\Theta(t, \epsilon) \\
C(t, \epsilon)
\end{array}\right)=\sum_{n} \epsilon^{n}\left(\begin{array}{c}
W^{(n)}(t) \\
\Theta^{(n)}(t) \\
C^{(n)}(t)
\end{array}\right)
$$

and the expansion is inserted into the governing equations, which are examined at each order in $\epsilon$.

The zero-th order system describes the unmodulated state, whose marginally-stable solution is given by the above eigenmode. The first order system describes the response to the small-amplitude sinusoidal forcing with frequency $\omega$. The solution can be written in the 
form

$$
\left(\begin{array}{c}
W^{(1)}(t) \\
\Theta^{(1)}(t) \\
C^{(1)}(t)
\end{array}\right)=\left(\begin{array}{c}
W_{1} \\
\Theta_{1} \\
C_{1}
\end{array}\right) e^{i \omega t}+\text { c.c. }
$$

where 'c.c.' denotes the complex conjugate of the preceding term. Here the coefficients are complex constants given by

$$
\begin{gathered}
W_{1}=\frac{a^{2}(\mathrm{Rs}-\mathrm{Ra})}{2\left(\pi^{2}+a^{2}\right)}\left[\left(\pi^{2}+a^{2}\right)^{2}+\frac{a^{2} \mathrm{Ra}}{\left(\pi^{2}+a^{2}+i \omega \mathrm{Pr}\right)}-\frac{a^{2} \mathrm{Rs}}{\left(\pi^{2}+a^{2}+i \omega \mathrm{Sc}\right)}+i \omega\left(\pi^{2}+a^{2}\right)\right]^{-1} \\
\Theta_{1}=\frac{-\operatorname{Pr} W_{1}}{\left(\pi^{2}+a^{2}+i \omega \mathrm{Pr}\right)}
\end{gathered}
$$

and

$$
C_{1}=\frac{-\mathrm{Sc} W_{1}}{\left(\pi^{2}+a^{2}+i \omega \mathrm{Sc}\right)}
$$

Through first order, the system has a time-periodic response to the driving force for any frequency $\omega$. At second order, the system describes the response to harmonics generated by the interaction of the sinusoidal forcing with the first-order response. A non-periodic, secular response is generated by the resulting mean component of the forcing unless a solvability condition on $\omega$ is satisfied. The system at second order has the form

$$
\begin{aligned}
\left(\pi^{2}+a^{2}\right) \frac{d W^{(2)}}{d t}+\left(\pi^{2}+a^{2}\right)^{2} W^{(2)}-a^{2} \operatorname{Ra} \frac{\Theta^{(2)}}{\operatorname{Pr}}+a^{2} \operatorname{Rs} \frac{C^{(2)}}{\mathrm{Sc}}=a^{2} \operatorname{Ra} \frac{\Theta^{(1)}}{\operatorname{Pr}} \cos \omega t-a^{2} \mathrm{Rs} \frac{C^{(1)}}{\mathrm{Sc}} \cos \omega t \\
\frac{d \Theta^{(2)}}{d t}+\left(\pi^{2}+a^{2}\right) \frac{\Theta^{(2)}}{\operatorname{Pr}}+W^{(2)}=0 \\
\frac{d C^{(2)}}{d t}+\left(\pi^{2}+a^{2}\right) \frac{C^{(2)}}{\mathrm{Sc}}+W^{(2)}=0 .
\end{aligned}
$$

Setting the mean component of the right hand side to zero gives

$$
0=\left\{\frac{\mathrm{Rs} W_{1}}{\left(\pi^{2}+a^{2}+i \omega \mathrm{Sc}\right)}-\frac{\mathrm{Ra} W_{1}}{\left(\pi^{2}+a^{2}+i \omega \mathrm{Pr}\right)}\right\}+c . c,
$$

which is an equation for the value of $\omega=\omega_{0}$ that allows the periodic solution to exist along the separatrix curve near $\epsilon=0$. This equation is equivalent to a quartic polynomial in $\omega_{0}^{2}$, whose roots may be found by inspection; there is a trivial root and two negative roots given by $\omega_{0}^{2}=-\left(\pi^{2}+a^{2}\right)^{2} / \operatorname{Pr}^{2}$ and $\omega_{0}^{2}=-\left(\pi^{2}+a^{2}\right)^{2} / \mathrm{Sc}^{2}$. The only root which can be positive is given by the expression

$$
\omega_{0}^{2}=\frac{a^{2}\left\{(\mathrm{RsSc}-\mathrm{RaPr})\left(\pi^{2}+a^{2}\right)^{3} / a^{2}+\mathrm{RsRa}(\mathrm{Sc}-\mathrm{Pr})^{2}\right\}}{\left(\pi^{2}+a^{2}\right) \mathrm{Pr}^{2} \mathrm{Sc}^{2} N^{2}}
$$

where $N$ is the Brunt-Väisälä frequency given above in (11). 
For the parameters under consideration, this equation gives the value $\omega_{0}=76.639$, or $1 / \omega_{0}=0.01305$, as shown in Fig. 6. By introducing an expansion $\omega=\omega_{0}+\sum \epsilon^{n} \omega^{(n)}$ for the frequency as well, this procedure could be continued to higher order in $\epsilon$ to obtain the curvature of the separatrix at $\epsilon=0$, but we have not pursued this question. In the language of bifurcation theory, the effect of detuning the system by taking Rs slightly different than $\mathrm{Rs}^{*}$ has the nature of an imperfection [35], and could be analyzed systematically as well.

The occurrence of the separatrix terminating at the frequency $\omega_{0}$ is a feature of the double-diffusive system; this phenomenon does not occur in the single-component case (c.f. Ref. [20], p. 210, for example). As Ra becomes small, the unstable region bounded on the right by the separatrix, and bounded below by the interval from zero to $1 / \omega_{0}$, shrinks in size and disappears as $N \rightarrow 0$; this behavior is indicated by the values of $1 / \omega_{0}$ shown in Table II for $\operatorname{Pr}=0.1$ and $\mathrm{Sc}=10$. The dependence of $\omega_{0}$ on $\mathrm{Ra}$ is not monotonic; the largest value of $1 / \omega_{0}$ occurs for $\mathrm{Ra} \approx 250$, and $1 / \omega_{0}$ tends to zero for smaller values of $\mathrm{Ra}$.

Note that the use of the term "fundamental mode" is really motivated by the instability of the modulated system for $\mathrm{Rs}>\mathrm{Rs}^{*}$ at low values of $\epsilon$ such as illustrated in Fig. 4. For the double-diffusive case with moderate values of Ra, Fig. 5 illustrates that this "fundamental mode" becomes a synchronous lobe for $R s<\mathrm{Rs}^{*}$, and in appearance is not dissimilar to the other lobes which are commonly termed "resonant modes" in the literature. Moreover, as illustrated in Fig. 3, the "fundamental mode" also persists as a synchronous lobe in the case $\delta=0$ in which the mean component of the gravitation vanishes. This mode thus can exist in situations which are quite removed from the more familiar setting in which the base state is marginally stable in the absence of modulation $(\epsilon=0)$.

\subsection{Diffusive Regime}

For our calculations in the diffusive regime, where oscillatory onset can occur in the absence of modulation, we choose parameters such that this is the case (i.e., steady onset is also possible in this regime, see Fig. 1). For the same values of the Prandtl and Schmidt numbers $(\operatorname{Pr}=0.1, \mathrm{Sc}=10)$ and the wavenumber $(a=\pi / \sqrt{2})$ used for the calculations in the fingering regime, we select a value of $\mathrm{Rs}=-300000$ which yields a critical $\mathrm{Ra}$ of approximately the same magnitude as the previous cases. For these parameter values, (9) yields a critical value of $\mathrm{Ra}=-1030.5$. The corresponding oscillatory frequency is $\sigma_{i}=95$ from $(10)$.

Figs. 7 and 8 show the stability diagrams ( $\epsilon$ versus $1 / \omega$ ) for sinusoidal modulation in the presence of steady acceleration $(\delta=1)$ for a subcritical value of $R a=-1000$ and a supercritical value of $\mathrm{Ra}=-1051$, respectively. Both figures consist of regions of resonant instability where the behavior alternates between subharmonic and synchronous modes as illustrated by the vertical and horizontal shading lines, respectively. The resonant bands are similar for the two values of Ra, except for the minima. Figs. 7 and 8 differ substantially in the unstable crosshatched region where instability occurs by complex conjugate pairs. In Fig. 8, the magnitude of $\mathrm{Ra}$ lies above the unmodulated critical value and this produces a fundamental instability which corresponds to the crosshatched region. Again, the usage of the term "fundamental instability" is intended to suggest the relation of this mode to the associated instability of the base state in the absence of modulation $(\epsilon=0)$; in the diffusive 
regime the unmodulated instability occurs via complex conjugate pairs for $\sigma_{i}$.

There is a similarity in the behavior of the fundamental instability in the present diffusive case to that in the fingering case which was shown in Figs. 4 and 5. In both cases, for unmodulated subcritical conditions (Figs. 5 and 7), the fundamental mode appears as a lobe whose minimum decreases as the unmodulated system becomes less stable. In the diffusive case shown in Fig. 7, this lobe consists of a pair of complex conjugate growth rates. As the unmodulated system becomes unstable, the boundary of the lobe transforms into a separatrix curve marking the transition from unstable to stable in a similar manner to Fig. 6 . At the highest frequencies, the system is unable to react to the sinusoidal modulation, and the stability or instability of the region matches that of the unmodulated solution. As in the fingering case, an asymptotic analysis for $\epsilon<<1$ can be used to determine the values of the forcing frequency $\omega$ along the separatrix in the critical case $\mathrm{Ra}=\mathrm{Ra} \mathrm{a}^{*}$; details are given in Appendix I. For the case appropriate to Figs. 7 and 8 with $R s=-300000$ and $\mathrm{Ra}=\mathrm{Ra}^{*}=-1030.5$, the separatrix hits the axis $\epsilon=0$ at $1 / \omega^{(0)}=4.23 \cdot\left(10^{-3}\right)$. This value of $\omega$ for the separatrix is near but distinct from the point $\omega=2 \sigma_{i}$ where the resonant mode of highest frequency intersects the axis $\epsilon=0$. Further, as the magnitude of the solutal Rayleigh number is decreased, these two points diverge from each other, with the separatrix intersecting the axis $\epsilon=0$ at larger and larger values of $\omega$.

For the subcritical case, Fig. 7, the highest frequency resonant band has a ininimum of approximately $\epsilon=10^{-2}$ at $\omega \approx 190$, which is twice the unmodulated oscillatory frequency, $\sigma_{i}$. The minimum of the $m$ th resonant band occurs at $\omega \approx 2 \sigma_{i} / m$. As the modulation frequency decreases, the minimum amplitude of the resonant bands increases. For the supercritical case shown in Fig. 8, strong resonance is obtained for both $\omega=\sigma_{i}$ and $2 \sigma_{i}$ with synchronous and subharmonic response, respectively. There are regions in the resonant bands where the system is doubly unstable; this occurs in the vicinity of the transition from fundamental (complex conjugate modes) to resonant regions.

The penetration at low values of $\epsilon$ of the high-frequency subharmonic lobe appears to be a resonance phenomenon: if $R a$ is set to its unmodulated critical value $R a^{*}$, then for $\epsilon=0$ the system has a marginally-stable oscillatory mode with frequency $\sigma_{i}$ given by equation (10). The governing equations describing the first-order correction for the case of small modulation amplitude then have the possibility of resonance, since the interaction of the driving frequency $\omega$ with the zero-th order mode gives rise to inhomogeneous forcing terms with frequency $\omega \pm \sigma_{i}$. The frequency difference is thus in resonance for $\omega=2 \sigma_{i}$. This observation is developed in Appendix II, where expressions for the amplitudes $\epsilon$ of the marginal stability curves are given for $\left|\omega-2 \sigma_{i}\right| \ll 1$. Resonance at other forcing frequencies may also occur at higher orders; for example, at second order a resonance occurs when $\omega=\sigma_{i}$ (see Fig. 8).

To better understand the resonant behavior exhibited by the cases shown in Figs. 7 and 8 , we plot in Fig. 9 the stability region for the single component case obtained by setting $\mathrm{Ra}=$ 0 and keeping $R s=-300000$, which is a highly stable situation in the absence of modulation and subject only to resonant instability. The structure of the resonance bands is somewhat similar between the single component and thermosolutal cases; however, the minimum of the 
resonant bands is considerably broader and a much higher modulation amplitude is required for instability in the single component case. For $\mathrm{Ra}=0$, the governing equations reduce to the damped Mathieu equation

$$
C_{\tau \tau}+\frac{\left(\pi^{2}+a^{2}\right)}{\omega}\left(1+\frac{1}{\mathrm{Sc}}\right) C_{\tau}+\frac{1}{\omega^{2} \mathrm{Sc}}\left[\left(\pi^{2}+a^{2}\right)^{2}-\frac{a^{2} \mathrm{Rs}}{\left(\pi^{2}+a^{2}\right)}(1+\epsilon \cos \tau)\right] C=0
$$

where $\tau=\omega t$. Note that the damping term is proportional to $1 / \omega$; the damping is responsible for the increase in the minima of the lobes as the frequency decreases. If the damping is neglected in this equation, the resonant modes then extend down to zero modulation amplitude $(\epsilon=0)$, where the resonant driving frequencies of these undamped modes are given by the expressions $1 / \omega_{m}^{2}=m^{2} \mathrm{Sc}\left(\pi^{2}+a^{2}\right) /\left(4 a^{2}\{-\mathrm{Rs}\}\right)$ in the limit of large $|\mathrm{Rs}|$. For the conditions in Figs. 7 and 8 with $R s=-300000$, this gives a value $1 / \omega_{1}=5.0\left(10^{-3}\right)$, which is in good agreement with the observed value $1 / \omega \approx 5.27\left(10^{-3}\right)$ for the resonant mode with the highest frequency. While it is apparent in the thermosolutal case that the resonance is tuned to the unmodulated oscillation frequency $\sigma_{i}$, it is interesting that in the single component case there is only a small shift of the frequency of the minima from the thermosolutal case. The buoyancy frequency for this single component case is $1 / N=5.78\left(10^{-3}\right)$, which is in the vicinity of the highest frequency subharmonic minimum.

In Fig. 10 we plot the minimum values of $\epsilon$ on the highest frequency subharmonic mode (solid curve) and the highest frequency synchronous mode (dashed curve) for values of the thermal Rayleigh number ranging from $\mathrm{Ra}=\mathrm{Ra}^{*}$ down to $\mathrm{Ra}=0$; the variable on the horizontal axis is $\left(\mathrm{Ra}^{*}-\mathrm{Ra}\right) / \mathrm{Ra}^{*}$. The curves illustrate the relative rate of descent of these modes as the critical thermal Rayleigh number is approached and resonance occurs. The values for the subharmonic mode decrease linearly with $\left(\mathrm{Ra}^{*}-\mathrm{Ra}\right) / \mathrm{Ra}^{*}$, whereas the values for the synchronous mode show a square root dependence.

In Fig. 11 we consider thermosolutal convection for the same Rayleigh numbers as in Fig. 7 in the absence of background acceleration $(\delta=0)$, so that the system is stable for $\epsilon$ $=0$. Alternating bands of resonant instability are again obtained, although the structure is considerably different from that of Fig. 7. The onset of instability as $\epsilon$ is increased is a smooth function of frequency and does not exhibit the cusps of Fig. 7. The vertical scale of Fig. 11 has been increased in order to show the region of complex conjugate instability, which occurs at high frequencies in both cases. For various ranges of frequencies, particularly at low frequencies, the $\delta=0$ case is less stable than the $\delta=1$ case. When $\delta=0$, the same results would be obtained if the sign of both Rayleigh numbers was changed. During half of the modulation cycle the thermal field is destabilizing, while for the other half the solute field is destabilizing. Since the magnitude of the solutal Rayleigh number is very large, the solutal instability dominates, and similar results would be obtained if the thermal Rayleigh number was set to zero, although the complex conjugate mode of instability only occurs for the thermosolutal case.

The single component case with $\delta=0$ was previously described in Fig. 2 in terms of the thermal field. To the extent that the thermal Rayleigh number can be neglected for the case in Fig. 11, the results are identical to those of Fig. 2 with the appropriate rescaling of $\epsilon$ and 
$\omega$. The $\omega$ for the solutal case is smaller by a factor of $10(\sqrt{\mathrm{Sc} / \mathrm{Pr}})$ and $\epsilon$ is smaller by a factor of 300 . We note that the modulation amplitude in the single component problem does depend on the Prandtl (or Schmidt) number, but the dependence is of the form $\sqrt{\mathrm{Pr}_{\mathrm{r}}}+1 / \sqrt{\mathrm{Pr}_{\mathrm{r}}}$ [20], so that the thermal case with $\operatorname{Pr}=0.1$ is equivalent to the solutal case with $\mathrm{Sc}=10$.

\section{Discussion and Conclusions}

We have considered the effect of sinusoidal gravity modulation on the onset of thermosolutal convection in an infinite layer with stress-free boundaries; this reduces the problem to a set of three coupled ordinary differential equations which are solved numerically using Floquet theory. The problem still depends on a large number of parameters. The unmodulated problem depends on the thermal and solutal Rayleigh numbers, the Prandtl number and the Schmidt number, while the modulation is characterized by the dimensionless frequency and amplitude. We have only considered situations for which the fluid layer is stably stratified, but have treated both the "fingering" and "diffusive" regimes of double-diffusive convection. Calculations have been carried out both with and without steady background acceleration, with the latter case being relevant to microgravity conditions.

As in the case of a single component system, sinusoidal modulation can destabilize a mode that is stable in the unmodulated case, or stabilize an unstable mode, with the stability characteristics depending on the frequency of modulation and the degree to which the thermal and solutal Rayleigh numbers differ from the unmodulated critical values. For large frequencies the effect of modulation on stability decreases, so that the stability or instability of the mode matches the unmodulated case. In addition to synchronous and subharmonic response to the modulation frequency, which also occurs for the single component case, instability in the double-diffusive system can occur via a complex conjugate mode. In general, the frequency (imaginary part) of the conjugate mode is unrelated to the modulation frequency.

In the fingering regime, where the solutal field is destabilizing, sinusoidal modulation produces resonant lobes of instability that are relatively unaffected by the level of background acceleration. When the unmodulated system is unstable there is a fundamental region of instability having a synchronous response. However, in contrast to the single component case, at subcritical values this fundamental region remains and becomes lobe-like (see Fig. 6).

In the diffusive regime the thermal field is destabilizing and oscillatory onset occurs in the unmodulated case. With modulation the fundamental region of instability corresponds to a complex conjugate pair. The regions of resonant instability which occur exhibit strong coupling with the unmodulated oscillatory frequency, with sharp minima at multiples of this frequency.

In the presence of gravity modulation the two component system exhibits more complicated dynamical behavior. These calculations provide some insight into this behavior. In the practical application to directional solidification the behavior is further complicated since the solute and temperature act over different length scales and the solid-liquid interface is a free boundary. Further, the solidification problem cannot be reduced to ordinary differential equations, and for typical metallic alloys the Schmidt and Prandtl numbers differ by 
four orders of magnitude. Although calculations for directional solidification are in progress, these factors limit the investigation; whereas for the model presented here, a wide-range of conditions can be studied.

\section{Acknowledgements}

We wish to thank Y. Vega-Torres for performing some of the calculations. This work was conducted with the support of the Microgravity Science and Applications Division of the National Aeronautics and Space Administration and the Applied and Computational Mathematics Program of the Defense Advanced Research Projects Agency. 


\section{Appendix I}

Much as in the fingering case, in the diffusive case there is a fundamental mode which is unstable for small modulation amplitudes when the magnitude $\mathrm{Ra}$ exceeds that of the unmodulated critical value $\mathrm{Ra}^{*}$. In this case the fundamental mode is neither synchronous nor subharmonic, so that $\sigma_{i}$ assumes values other than zero and $\omega / 2$; this is associated with the instability of the unmodulated base state to oscillatory modes with non-zero $\sigma_{i}$. As Ra decreases in magnitude to values that are subcritical with respect to $\mathrm{Ra}^{*}$, the fundamental mode contracts to a region bounded by a separatrix for $\mathrm{Ra}=\mathrm{Ra}^{*}$, and then for still smaller magnitudes becomes a lobe located at higher frequencies; this behavior may be inferred by comparing Figs. 7 and 8. The resonant modes do not show large modifications as $\mathrm{Ra}$ is varied in the vicinity of $\mathrm{Ra}^{*}$ (see also Fig. 10 for the behavior of the first two resonant modes as $R a$ is varied).

The local behavior of the separatrix at small modulation amplitudes may again be described by an expansion procedure in $\epsilon$. In the diffusive case, however, the separatrix does not represent time-periodic marginally-stable solutions since $\sigma_{i}$ will generally be incommensurate with $\omega$, and the procedure used previously must be modified. The more complicated time-dependence is taken into account by representing the marginally-stable solutions in Floquet form as

$$
\left(\begin{array}{c}
W(t) \\
\Theta(t) \\
C(t)
\end{array}\right)=e^{i \sigma_{i} t}\left(\begin{array}{c}
\widehat{W}(t) \\
\widehat{\Theta}(t) \\
\widehat{C}(t)
\end{array}\right),
$$

where the frequency $\sigma_{i}$ is to be determined, and the variables $\widehat{W}(t), \widehat{\Theta}(t)$, and $\widehat{C}(t)$ are periodic with frequency $\omega$. The governing equations then have the form

$$
\begin{gathered}
\omega \frac{d \widehat{W}}{d \tau}+i \sigma_{i} \widehat{W}=-\left(\pi^{2}+a^{2}\right) \widehat{W}+\frac{a^{2} \mathrm{Ra}}{\left(\pi^{2}+a^{2}\right)}(1+\epsilon \cos \tau) \frac{\widehat{\Theta}}{\operatorname{Pr}}-\frac{a^{2} \mathrm{Rs}}{\left(\pi^{2}+a^{2}\right)}(1+\epsilon \cos \tau) \frac{\widehat{C}}{\mathrm{Sc}}, \\
\omega \frac{d \widehat{\Theta}}{d \tau}+i \sigma_{i} \widehat{\Theta}=-\left(\pi^{2}+a^{2}\right) \frac{\widehat{\Theta}}{\operatorname{Pr}}-\widehat{W}
\end{gathered}
$$

and

$$
\omega \frac{d \widehat{C}}{d \tau}+i \sigma_{i} \widehat{C}=-\left(\pi^{2}+a^{2}\right) \frac{\widehat{C}}{\mathrm{Sc}}-\widehat{W}
$$

where we have again written $\tau=\omega t$. For fixed $\mathrm{Rs}$ and $\mathrm{Ra}=\mathrm{Ra}^{*}$, we expand the variables in the form

$$
\left(\begin{array}{c}
\widehat{W}(\tau, \epsilon) \\
\widehat{\Theta}(\tau, \epsilon) \\
\widehat{C}(\tau, \epsilon) \\
\sigma_{i}(\epsilon)
\end{array}\right)=\sum_{n} \epsilon^{n}\left(\begin{array}{c}
W^{(n)}(\tau) \\
\Theta^{(n)}(\tau) \\
C^{(n)}(\tau) \\
\sigma_{i}^{(n)}
\end{array}\right),
$$

where the zero-th order solution corresponds to the unmodulated marginally stable stable solution, with $\sigma_{i}^{(0)}$ given by the expression (10); the limiting value $\omega^{(0)}$ of the forcing frequency along the separatrix as $\epsilon \rightarrow 0$ is to be found as part of the solution. Higher order corrections 
to $\omega=\omega(\epsilon)$ along the separatrix could be computed by an extension of the expansion, but as we will not pursue this point, it suffices for our purposes to determine only the zeroth order term $\omega^{(0)}$. We then have the leading order solution

$$
\left(\begin{array}{l}
W^{(0)}(\tau) \\
\Theta^{(0)}(\tau) \\
C^{(0)}(\tau)
\end{array}\right)=\left(\begin{array}{c}
1 \\
-\operatorname{Pr} /\left(\pi^{2}+a^{2}+i \sigma_{i} \mathrm{Pr}\right) \\
-\mathrm{Sc} /\left(\pi^{2}+a^{2}+i \sigma_{i} \mathrm{Sc}\right)
\end{array}\right)
$$

The first order solution satisfies $\sigma_{i}^{(1)}=0$ and

$$
\left(\begin{array}{c}
W^{(1)}(\tau) \\
\Theta^{(1)}(\tau) \\
C^{(1)}(\tau)
\end{array}\right)=e^{i \tau}\left(\begin{array}{c}
W_{1}^{(+)} \\
\Theta_{1}^{(+)} \\
C_{1}^{(+)}
\end{array}\right)+e^{-i \tau}\left(\begin{array}{c}
W_{1}^{(-)} \\
\Theta_{1}^{(-)} \\
C_{1}^{(-)}
\end{array}\right)
$$

where

$$
\begin{aligned}
& \Theta_{1}^{( \pm)}=\frac{-\operatorname{Pr} W_{1}^{( \pm)}}{\left(\pi^{2}+a^{2}+i\left[\sigma_{i}^{(0)} \pm \omega^{(0)}\right] \mathrm{Pr}\right)} \\
& C_{1}^{( \pm)}=\frac{-\mathrm{Sc} W_{1}^{( \pm)}}{\left(\pi^{2}+a^{2}+i\left[\sigma_{i}^{(0)} \pm \omega^{(0)}\right] \mathrm{Sc}\right)}
\end{aligned}
$$

and

$$
\begin{gathered}
{\left[\left(\pi^{2}+a^{2}\right)^{2}+i\left[\sigma_{i}^{(0)} \pm \omega\right]\left(\pi^{2}+a^{2}\right)-\frac{a^{2} \mathrm{Rs}}{\left(\pi^{2}+a^{2}+i\left[\sigma_{i}^{(0)} \pm \omega\right] \mathrm{Sc}\right)}+\right.} \\
\left.+\frac{a^{2} \mathrm{Ra}}{\left(\pi^{2}+a^{2}+i\left[\sigma_{i}^{(0)} \pm \omega\right] \mathrm{Pr}\right)}\right] W_{1}^{( \pm)}=\frac{a^{2}}{2}\left[\frac{\mathrm{Rs}}{\left(\pi^{2}+a^{2}+i \sigma^{(0)} \mathrm{Sc}\right)}-\frac{\mathrm{Ra}}{\left(\pi^{2}+a^{2}+i \sigma^{(0)} \mathrm{Pr}\right)}\right] .
\end{gathered}
$$

The second-order system can be written in the form

$$
\omega \frac{d}{d \tau}\left(\begin{array}{l}
W^{(2)}(\tau) \\
\Theta^{(2)}(\tau) \\
C^{(2)}(\tau)
\end{array}\right)=A\left(\begin{array}{l}
W^{(2)}(\tau) \\
\Theta^{(2)}(\tau) \\
C^{(2)}(\tau)
\end{array}\right)+b(\tau)
$$

where the time-independent matrix $A$ is given by

$$
A=\left(\begin{array}{ccc}
-\left(\pi^{2}+a^{2}+i \sigma_{i}^{(0)}\right) & a^{2} \operatorname{Ra}\left[\operatorname{Pr}\left(\pi^{2}+a^{2}\right)\right]^{-1} & -a^{2} \operatorname{Rs}\left[\operatorname{Sc}\left(\pi^{2}+a^{2}\right)\right]^{-1} \\
-1 & -\left(\pi^{2}+a^{2}+i \sigma_{i}^{(0)} \operatorname{Pr}\right) / \operatorname{Pr} & 0 \\
-1 & 0 & -\left(\pi^{2}+a^{2}+i \sigma_{i}^{(0)} \mathrm{Sc}\right) / \mathrm{Sc}
\end{array}\right)
$$

and the vector $b(\tau)$ is given by

$$
b(\tau)=-i \sigma_{i}^{(2)}\left(\begin{array}{l}
W^{(0)} \\
\Theta^{(0)} \\
C^{(0)}
\end{array}\right)+\frac{a^{2}}{\left(\pi^{2}+a^{2}\right)}\left(\begin{array}{c}
(\mathrm{Ra} / \operatorname{Pr}) \Theta^{(1)} \cos \tau-(\mathrm{Rs} / \mathrm{Sc}) C^{(1)} \cos \tau \\
0 \\
0
\end{array}\right)
$$


We now can determine $\sigma_{i}^{(2)}$ and $\omega=\omega^{(0)}$ from a solvability condition by requiring the mean component of $b(\tau)$ to be orthogonal to the null vector $\eta$ of the adjoint of $A$, where

$$
\eta=\left(\begin{array}{c}
1 \\
a^{2} \operatorname{Ra}\left[\left(\pi^{2}+a^{2}\right)\left(\pi^{2}+a^{2}-i \sigma_{i}^{(0)} \operatorname{Pr}\right)\right]^{-1} \\
-a^{2} \operatorname{Rs}\left[\left(\pi^{2}+a^{2}\right)\left(\pi^{2}+a^{2}-i \sigma_{i}^{(0)} \mathrm{Sc}\right)\right]^{-1}
\end{array}\right) .
$$

The mean component of $b(\tau)$ is given in the expression

$$
\begin{aligned}
& \frac{\omega}{2 \pi} \int_{0}^{2 \pi / \omega} b(\tau) d \tau=-i \sigma_{i}^{(2)}\left(\begin{array}{c}
1 \\
-\operatorname{Pr} /\left(\pi^{2}+a^{2}+i \sigma_{i}^{(0)} \operatorname{Pr}\right) \\
-\operatorname{Sc} /\left(\pi^{2}+a^{2}+i \sigma_{i}^{(0)} \mathrm{Sc}\right)
\end{array}\right)+ \\
& +\frac{a^{2}}{2\left(\pi^{2}+a^{2}\right)}\left(\begin{array}{c}
(\mathrm{Ra} / \mathrm{Pr})\left[\Theta_{1}^{+}+\Theta_{1}^{-}\right]-(\mathrm{Rs} / \mathrm{Sc})\left[C_{1}^{+}+C_{1}^{-}\right] \\
0 \\
0
\end{array}\right)
\end{aligned}
$$

the solvability condition has the form

$$
\begin{gathered}
i \sigma_{i}^{(2)}\left\{1+\frac{a^{2} \mathrm{RsSc}}{\left(\pi^{2}+a^{2}\right)\left(\pi^{2}+a^{2}+i \sigma_{i}^{(0)} \mathrm{Sc}\right)^{2}}-\frac{a^{2} \operatorname{RaPr}}{\left(\pi^{2}+a^{2}\right)\left(\pi^{2}+a^{2}+i \sigma_{i}^{(0)} \mathrm{Pr}\right)^{2}}\right\}= \\
\frac{a^{2}}{2\left(\pi^{2}+a^{2}\right)}\left\{\frac{\mathrm{Ra}}{\operatorname{Pr}}\left[\Theta_{1}^{+}+\Theta_{1}^{-}\right]-\frac{\mathrm{Rs}}{\mathrm{Sc}}\left[C_{1}^{+}+C_{1}^{-}\right]\right\} .
\end{gathered}
$$

This complex-valued expression provides two real equations in the two unknowns $\omega^{(0)}$ and $\sigma_{i}^{(2)}$, and some numerical solutions are given in Table III for various values of the solutal Rayleigh number $\mathrm{Rs}$ with $\mathrm{Sc}=10$ and $\operatorname{Pr}=0.1$. Also shown are the values of $\sigma_{i}^{(0)}$ for these cases as well. 


\section{Appendix II}

In the diffusive regime, the marginal stability curves of the resonant modes for $\mathrm{Ra}=\mathrm{Ra}$ *, $\omega \sim 2 \sigma_{i}$, and $\epsilon<<1$ may be described by an expansion procedure. To do this, it is convenient to rescale time by setting $\tau=\omega t$, and expanding the variables as

$$
\left(\begin{array}{c}
W(\tau, \epsilon) \\
\Theta(\tau, \epsilon) \\
C(\tau, \epsilon) \\
\omega(\epsilon)
\end{array}\right)=\sum_{n} \epsilon^{n}\left(\begin{array}{c}
W^{(n)}(\tau) \\
\Theta^{(n)}(\tau) \\
C^{(n)}(\tau) \\
\omega^{(n)}
\end{array}\right)
$$

where $\omega^{(0)}=2 \sigma_{i}$ and

$$
\left(\begin{array}{l}
W^{(0)}(\tau) \\
\Theta^{(0)}(\tau) \\
C^{(0)}(\tau)
\end{array}\right)=e^{i \alpha}\left(\begin{array}{c}
1 \\
-\operatorname{Pr} /\left(\pi^{2}+a^{2}+i \sigma_{i} \operatorname{Pr}\right) \\
-\mathrm{Sc} /\left(\pi^{2}+a^{2}+i \sigma_{i} \mathrm{Sc}\right)
\end{array}\right) e^{i \tau / 2}+\text { c.c. }
$$

is the unmodulated eigenfunction; here the appropriate phase $\alpha$ is to be determined as part of the solution procedure. The first order equations have the form

$$
\omega^{(0)} \frac{d}{d \tau}\left(\begin{array}{c}
W^{(1)}(\tau) \\
\Theta^{(1)}(\tau) \\
C^{(1)}(\tau)
\end{array}\right)=A\left(\begin{array}{c}
W^{(1)}(\tau) \\
\Theta^{(1)}(\tau) \\
C^{(1)}(\tau)
\end{array}\right)+b(\tau)
$$

where the time-independent matrix $A$ is given by

$$
A=\left(\begin{array}{ccc}
-\left(\pi^{2}+a^{2}\right) & a^{2} \operatorname{Ra}\left[\operatorname{Pr}\left(\pi^{2}+a^{2}\right)\right]^{-1} & -a^{2} \operatorname{Rs}\left[\operatorname{Sc}\left(\pi^{2}+a^{2}\right)\right]^{-1} \\
-1 & -\left(\pi^{2}+a^{2}\right) / \operatorname{Pr} & 0 \\
-1 & 0 & -\left(\pi^{2}+a^{2}\right) / \mathrm{Sc}
\end{array}\right)
$$

and the vector $b(\tau)$ is given by

$$
\begin{gathered}
b(\tau)=-\omega^{(1)}\left(\begin{array}{c}
W_{\tau}^{(0)} \\
\Theta_{\tau}^{(0)} \\
C_{\tau}^{(0)}
\end{array}\right)+\frac{a^{2}}{\left(\pi^{2}+a^{2}\right)}\left(\begin{array}{c}
(\mathrm{Ra} / \operatorname{Pr}) \Theta^{(0)} \cos \tau-(\mathrm{Rs} / \mathrm{Sc}) C^{(0)} \cos \tau \\
0 \\
0
\end{array}\right), \\
=\frac{-i \omega^{(1)}}{2} e^{i \alpha}\left(\begin{array}{c}
1 \\
-\operatorname{Pr} /\left(\pi^{2}+a^{2}+i \sigma_{i} \operatorname{Pr}\right) \\
-\mathrm{Sc} /\left(\pi^{2}+a^{2}+i \sigma_{i} \mathrm{Sc}\right)
\end{array}\right) e^{i \tau / 2}+\text { c.c. }+ \\
+\frac{a^{2}}{2\left(\pi^{2}+a^{2}\right)}\left(\begin{array}{c}
\mathrm{Rs} /\left(\pi^{2}+a^{2}-i \sigma_{i} \mathrm{Sc}\right)-\mathrm{Ra} /\left(\pi^{2}+a^{2}-i \sigma_{i} \mathrm{Pr}\right) \\
0 \\
0
\end{array}\right) e^{i \tau / 2}+\text { c.c. }+ \text { other harmonics. }
\end{gathered}
$$

A solvability condition for the existence of time-periodic solutions to these equations is that the vector coefficient of $e^{i \tau / 2}$ in the above expression for $b(\tau)$ must be orthogonal to the left eigenvector of $A$ corresponding to the eigenvalue $+i \sigma_{i}$. A similar condition in terms of 
the coefficient of $e^{-i \tau / 2}$ and the left eigenvector with eigenvalue $-i \sigma_{i}$ also holds; the two conditions are related through complex conjugation. The left eigenvector with eigenvalue $i \sigma_{i}$ satisfies $A^{T} \eta=-i \sigma_{i} \eta$, and is given by

$$
\eta=\left(\begin{array}{c}
1 \\
a^{2} \operatorname{Ra}\left[\left(\pi^{2}+a^{2}\right)\left(\pi^{2}+a^{2}-i \sigma_{i} \operatorname{Pr}\right)\right]^{-1} \\
-a^{2} \operatorname{Rs}\left[\left(\pi^{2}+a^{2}\right)\left(\pi^{2}+a^{2}-i \sigma_{i} \mathrm{Sc}\right)\right]^{-1}
\end{array}\right)
$$

The solvability condition has the form

$$
\begin{gathered}
\frac{a^{2}}{2\left(\pi^{2}+a^{2}\right)}\left\{\frac{\mathrm{Rs}}{\left(\pi^{2}+a^{2}-i \sigma_{i} \mathrm{Sc}\right)}-\frac{\mathrm{Ra}}{\left(\pi^{2}+a^{2}-i \sigma_{i} \mathrm{Pr}\right)}\right\}- \\
-\frac{i \omega^{(1)}}{2} e^{i \alpha}\left\{1-\frac{a^{2} \operatorname{Ra} \operatorname{Pr}}{\left(\pi^{2}+a^{2}\right)\left(\pi^{2}+a^{2}+i \sigma_{i} \operatorname{Pr}\right)^{2}}+\frac{a^{2} \mathrm{RsSc}}{\left(\pi^{2}+a^{2}\right)\left(\pi^{2}+a^{2}+i \sigma_{i} \mathrm{Sc}\right)^{2}}\right\}=0 .
\end{gathered}
$$

If we write this expression in the form

$$
\omega^{(1)} e^{i \alpha}=\beta e^{i \phi},
$$

then there are two solutions, one with $\omega^{(1)}=\beta$ and $\alpha=\phi$, and the other with $\omega^{(1)}=-\beta$ and $\alpha=\phi+\pi / 2$. Evaluating these quantities for the case $\mathrm{Rs}=-300000, \operatorname{Pr}=0.1$, and $\mathrm{Sc}=10.0$ gives $\beta=44.4876$ and $\phi=0.1855$. For $\epsilon=0.01$, this approximation predicts a frequency of $\omega^{(0)}+\epsilon \omega^{(1)}=190.1584$ on one branch of the marginal curve, which agrees with the corresponding numerical solution to six figures. 


\section{Table I}

Definition of Symbols

\begin{tabular}{lll}
\hline \multicolumn{2}{c}{ Dimensional Parameters } & \\
solute diffusion coefficient & $D$ & $\mathrm{~cm}^{2} / \mathrm{s}$ \\
thermal diffusion coefficient & $\kappa$ & $\mathrm{cm}^{2} / \mathrm{s}$ \\
thermal expansion coefficient & $\alpha$ & $\mathrm{K}^{-1}$ \\
solutal expansion coefficient & $\beta$ & $\mathrm{wt} \%^{-1}$ \\
kinematic viscosity & $\nu$ & $\mathrm{cm}^{2} / \mathrm{s}$ \\
thickness of layer & $d$ & $\mathrm{~cm}$ \\
gravitational field & $g=-g(\delta+\epsilon \cos \Omega t) \hat{\boldsymbol{z}}$ & $\mathrm{cm} / \mathrm{s}^{2}$ \\
reference gravitational acceleration & $g$ & $\mathrm{~cm} / \mathrm{s}^{2}$ \\
modulation frequency & $\Omega$ & $\mathrm{s}^{-1}$
\end{tabular}

\section{Dimensionless Parameters}

relative amplitude of steady component of acceleration

relative amplitude of modulated component of acceleration

Prandtl number

Schmidt number

thermal Rayleigh number

solutal Rayleigh number

dimensionless modulation frequency

$\delta$
$\epsilon$
$\mathrm{Pr}=\nu / \kappa$
$\mathrm{Sc}=\nu / D$
$\mathrm{Ra}=g \alpha \Delta T d^{3} /(\nu \kappa)$
$\mathrm{Rs}=g \beta \Delta c d^{3} /(\nu D)$
$\omega=\Omega d^{2} / \nu$




\section{Table II}

Separatrix for Fingering Case

\begin{tabular}{ccc}
\hline $\mathrm{Ra}$ & $\omega_{0}$ & $1 / \omega_{0} \times 10^{2}$ \\
10,000 & 188.2 & 0.531 \\
5,000 & 137.6 & 0.727 \\
2,000 & 95.4 & 1.049 \\
1,000 & 76.6 & 1.305 \\
500 & 66.2 & 1.510 \\
250 & 62.4 & 1.601 \\
100 & 66.9 & 1.495 \\
50 & 79.4 & 1.260 \\
20 & 120.7 & 0.828 \\
10 & 224.8 & 0.445 \\
7 & 673.2 & 0.149 \\
6.642 & $\infty$ & 0
\end{tabular}




\section{Table III}

Separatrix for Diffusive Case

\begin{tabular}{cccccc}
\hline$-\mathrm{Rs}$ & $-\mathrm{Ra}$ & $\sigma_{i}^{(0)}$ & $\omega_{0}$ & $1 / \omega_{0} \times 10^{3}$ & $\sigma_{i}^{(2)} \times 10^{2}$ \\
300000. & 1030.495 & 94.8568 & 236.33 & 4.23133 & 887.22 \\
250000. & 980.495 & 86.5899 & 245.42 & 4.07461 & 419.96 \\
200000. & 930.495 & 77.4455 & 263.74 & 3.79163 & 181.45 \\
150000. & 880.495 & 67.0657 & 307.59 & 3.25107 & 57.110 \\
100000. & 830.495 & 54.7522 & 510.55 & 1.95869 & 4.34146 \\
90000. & 820.495 & 51.9404 & 722.12 & 1.38481 & 0.970315
\end{tabular}




\section{References}

[1] H. Stommel, A.B. Arons, and D. Blanchard, Deep-Sea Res. 3, 152 (1956).

[2] J.S. Turner, Buoyancy Effects in Fluids (Cambridge U. P., New York, 1973).

[3] J.S. Turner, Ann. Rev. Fluid Mech. 6, 37 (1974).

[4] H.E. Huppert and J.S. Turner, J. Fluid Mech. 106, 299 (1981).

[5] C.F. Chen and D.H. Johnson, J. Fluid Mech. 138, 405 (1984).

[6] S. R. Coriell, M. R. Cordes, W. J. Boettinger, and R. F. Sekerka, J. Crystal Growth $49,13(1980)$.

[7] D. T. J. Hurle, E. Jakeman, and A. A. Wheeler, J. Crystal Growth 58, 163 (1982).

[8] D. T. J. Hurle, E. Jakeman, and A. A. Wheeler, Phys. Fluids 26, 624 (1983).

[9] R. J. Schaefer and S. R. Coriell, Met. Trans. 15 A, 2109 (1984).

[10] B. Caroli, C. Caroli, C. Misbah, and B. Roulet, J. Phys. (Paris) 46, 401 (1985).

[11] B. Caroli, C. Caroli, C. Misbah, and B. Roulet, J. Phys. (Paris) 46, 1657 (1985).

[12] D. R. Jenkins, J. Crystal Growth 102, 481 (1990).

[13] S. H. Davis, J. Fluid Mech. 212, 241 (1990).

[14] W. Knabe and D. Eilers, Acta Astronautica, 9, 187 (1982).

[15] J.I.D. Alexander and C.A. Lundquist, AIAA Journal 26, 34 (1988).

[16] J.I.D. Alexander, Microgravity Science and Technology 3, 2 (1990).

[17] W.S. Liu, M.F. Wolf, D. Elwell and R.S. Feigelson, J. Crystal Growth 82, 589 (1987).

[18] Y.-C. Lu, J.-J. Shiau, R.S. Feigelson and R. K. Route, J. Crystal Growth 102, 807 (1990).

[19] E. V. Zharikov, L. V. Prohod'ko, and N. R. Storozhev, J. Crystal Growth 99, 910 (1990).

[20] G. Z. Gershuni and E. M. Zhukhovitskii, Convective Stability of Incompressible Fluids (Keter., Jerusalem, 1976), Ch. 8.

[21] P. M. Gresho and R. L. Sani, J. Fluid Mech. 40, 783 (1970).

[22] S. Biringen and G. Danabasoglu, J. Thermophys. 4, 357 (1990). 
[23] S. Biringen and L.J. Peltier, Phys. Fluids A 2, 754 (1990).

[24] G. Venezian, J. Fluid Mech. 35, 243 (1969).

[25] S. H. Davis, Ann. Rev. Fluid Mech. 8, 57 (1976).

[26] R. G. Finucane and R. E. Kelly, Int. J. Heat Mass Transfer 19, 71 (1976).

[27] S. Ostrach, Ann. Rev. Fluid Mech. 14, 313 (1982).

[28] M. Wadih and B. Roux, J. Fluid Mech. 193, 391 (1988).

[29] G. Z. Gershuni, E. M. Zhukhovitskii, A. K. Kolesnikov, and Y. S. Yurkov, Int. J. Heat Mass Transfer 32, 2319 (1989).

[30] B.T. Murray, S.R. Coriell, and G.B. McFadden, J. Crystal Growth 110, 713 (1990).

[31] S. Chandrasekhar, Hydrodynamic and Hydromagnetic Stability (Oxford University Press, Oxford, 1961), p. 20.

[32] V. A. Yakubovich and V. M. Starzhinskii, Linear Differential Equations with Periodic Coefficients (Wiley, New York, 1975).

[33] D. Kahaner, C. Moler, and S. Nash, Numerical Methods and Software (Prentice Hall, New Jersey, 1989), p. 295.

[34] A.A. Wheeler, G.B. McFadden, B.T. Murray, and S.R. Coriell, Physics of Fluids A, in press.

[35] L. Bauer, H.B. Keller, and E.L. Reiss, SIAM Rev. 17, 101 (1975). 


\section{Figure Captions}

Figure 1. Stability diagram for unmodulated thermosolutal convection.

Figure 2. The relative modulation amplitude $\epsilon$ at the onset of thermal convection versus the inverse of the frequency $1 / \omega$ for sinusoidal gravitational acceleration. Shading with vertical lines represents subharmonic instability and shading with horizontal lines a synchronous instability.

Figure 3. The relative modulation amplitude $\epsilon$ at the onset of thermosolutal convection versus the inverse of the frequency $1 / \omega$ for sinusoidal gravitational acceleration. Shading with vertical lines represents subharmonic response and shading with horizontal lines a synchronous response. Crosshatched lines indicate a doubly unstable region characterized by the presence of complex conjugate pairs in the solution sets for $\sigma$.

Figure 4. The relative modulation amplitude $\epsilon$ at the onset of thermosolutal convection versus the inverse of the frequency $1 / \omega$ for sinusoidal gravitational acceleration. Without modulation the system is unstable.

Figure 5. The relative modulation amplitude $\epsilon$ at the onset of thermosolutal convection versus the inverse of the frequency $1 / \omega$ for sinusoidal gravitational acceleration. Without modulation the system is stable.

Figure 6. Synchronous response to the onset of modulated thermosolutal convection for values of Rs above and below the critical value of 1657 .

Figure 7. The relative modulation amplitude $\epsilon$ at the onset of thermosolutal convection versus the inverse of the frequency $1 / \omega$ for sinusoidal gravitational acceleration. Vertical shading lines represent a subharmonic instability; horizontal shading lines represent a synchronous instability; diagonally intersecting shading lines indicate instability via a complex conjugate pair.

Figure 8. The relative modulation amplitude $\epsilon$ at the onset of thermosolutal convection versus the inverse of the frequency $1 / \omega$ for sinusoidal gravitational acceleration. Shaded regions are unstable.

Figure 9. The relative modulation amplitude $\epsilon$ at the onset of solutal convection versus the inverse of the frequency $1 / \omega$ for sinusoidal gravitational acceleration.

Figure 10. The minimum values of the relative modulation amplitude $\epsilon$ on the neutral stability curves for the highest frequency subharmonic mode (solid curve) and the highest frequency synchronous mode (dashed curve) for values of the thermal Rayleigh number ranging from $R a=R a^{*}$ down to $R a=0$.

Figure 11. The relative modulation amplitude $\epsilon$ at the onset of thermosolutal convection versus the inverse of the frequency $1 / \omega$ for sinusoidal gravitational acceleration in the absence of background acceleration. Shaded regions are unstable. 

Unmodulated Thermosolutal Convection

$$
\operatorname{Pr}=1 \quad \mathrm{Sc}=10
$$

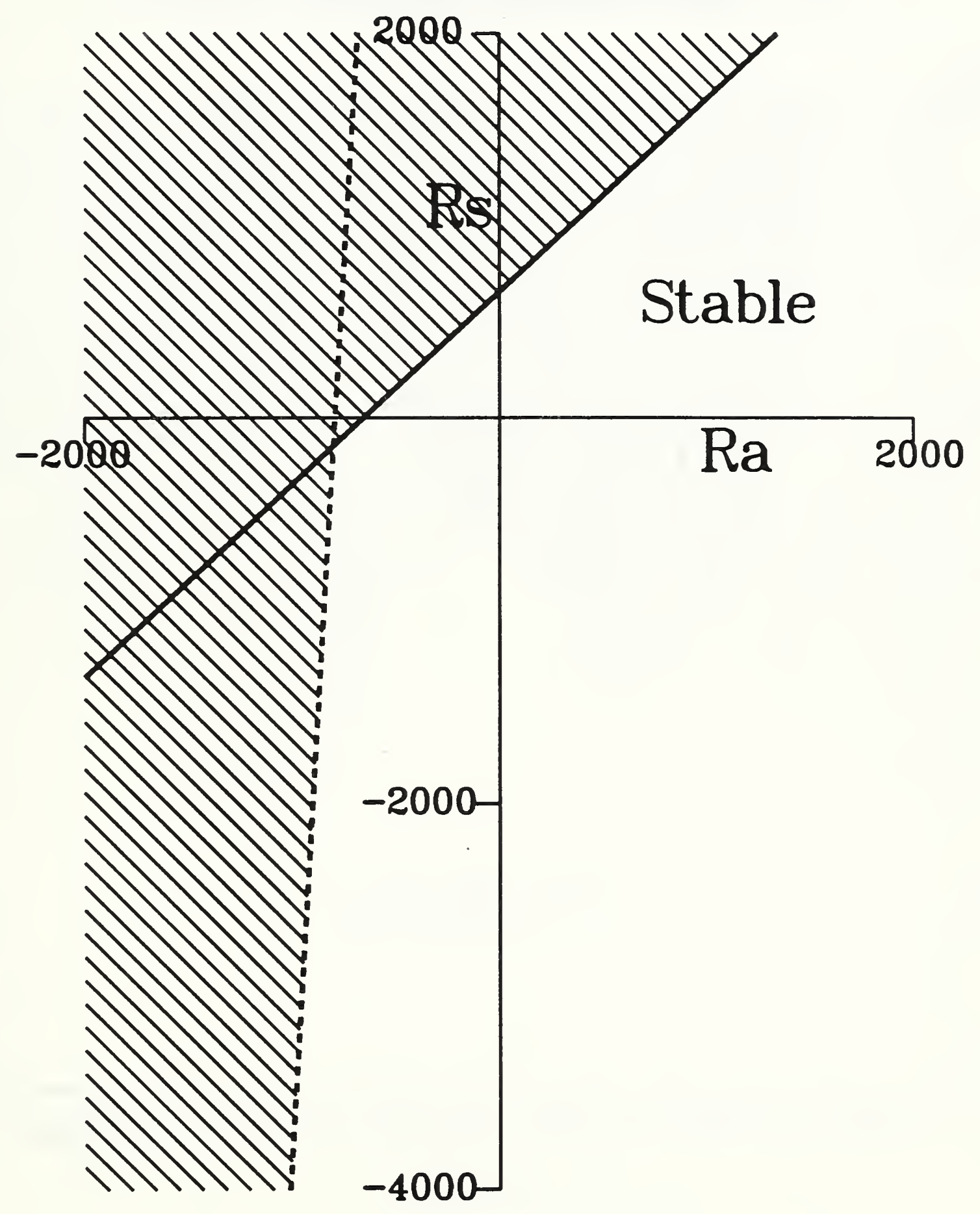


Modulated Thermal Convection

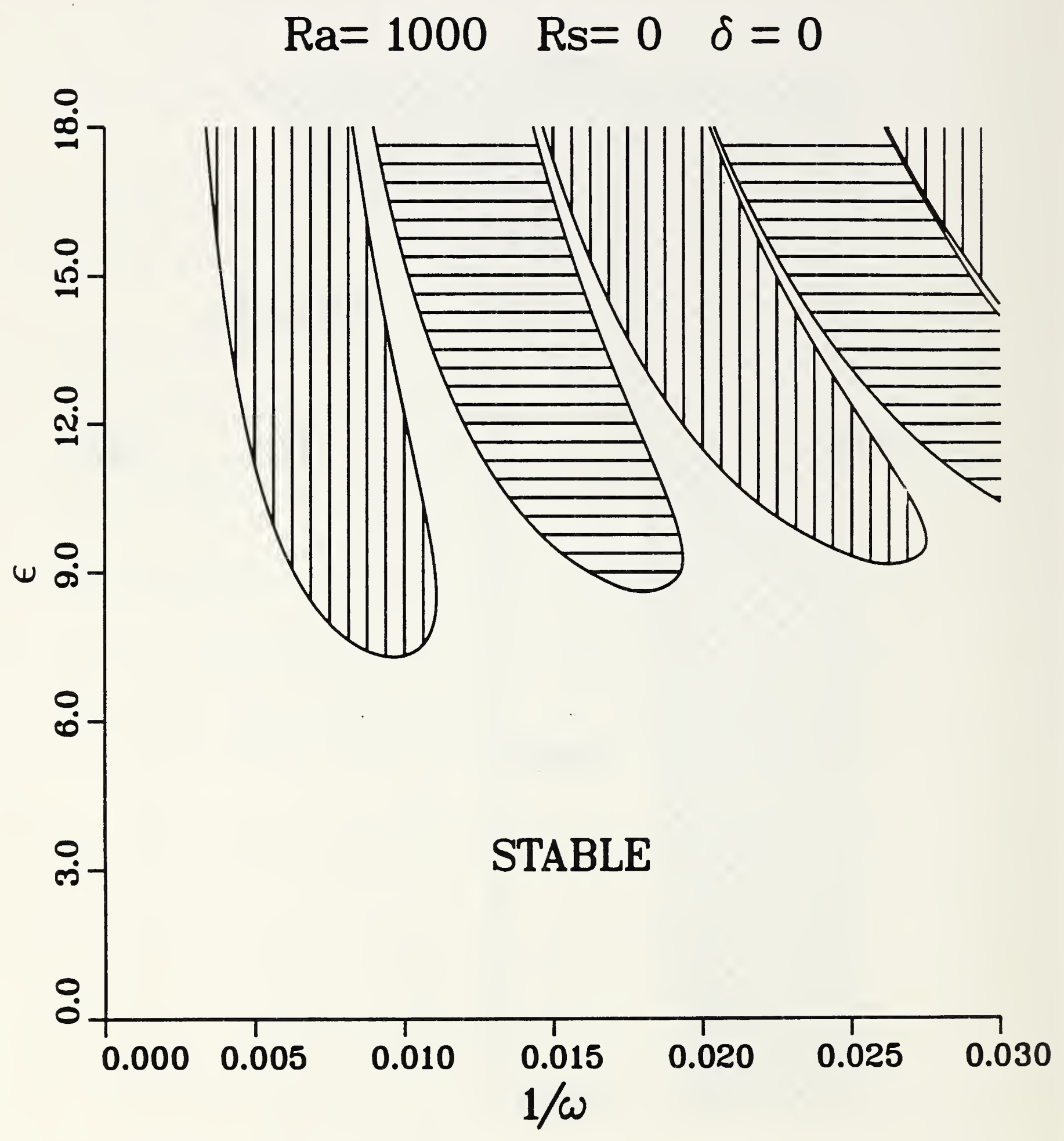

Figure 2 
Modulated Thermosolutal Convection

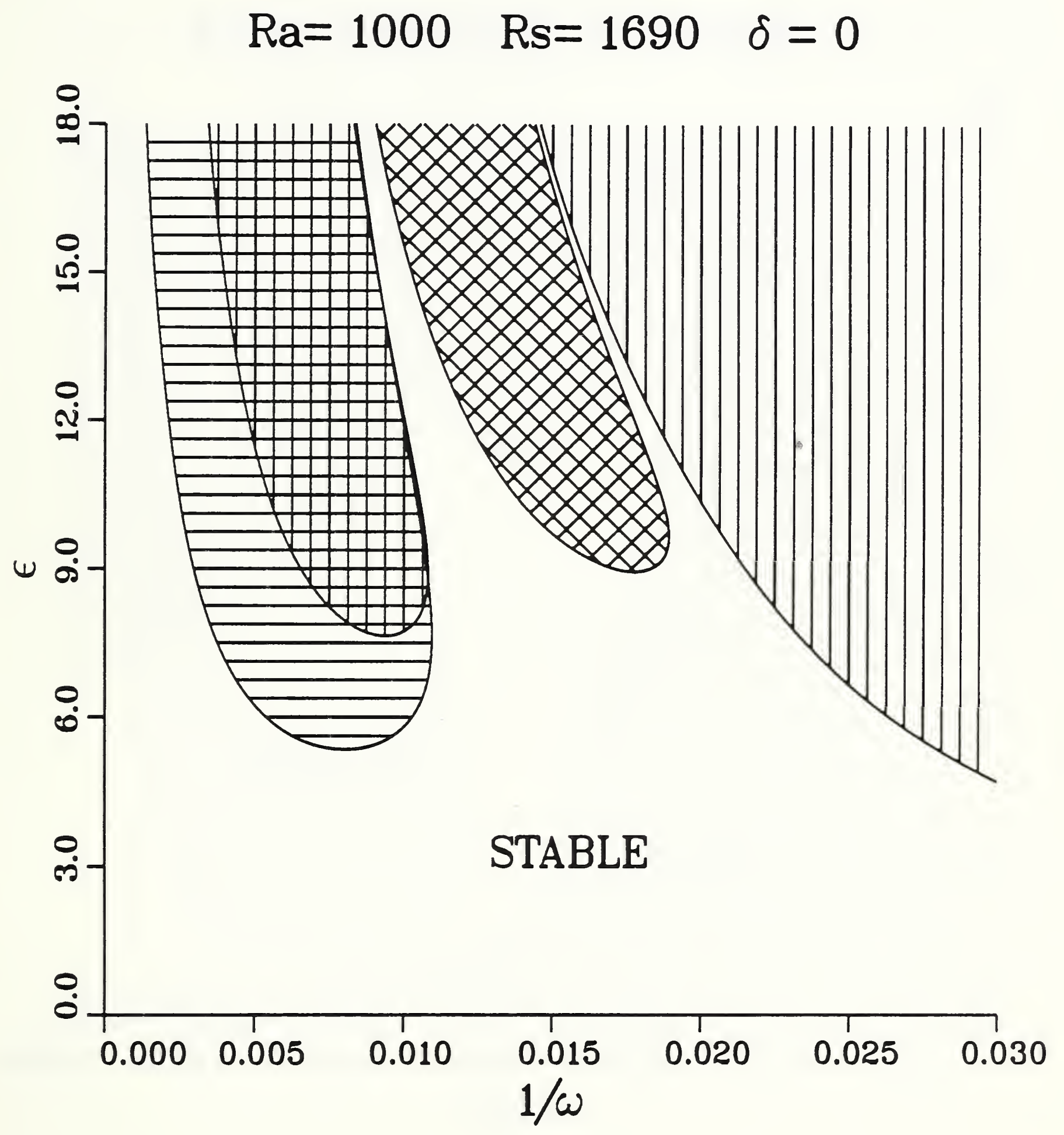




\section{Modulated Thermosolutal Convection}

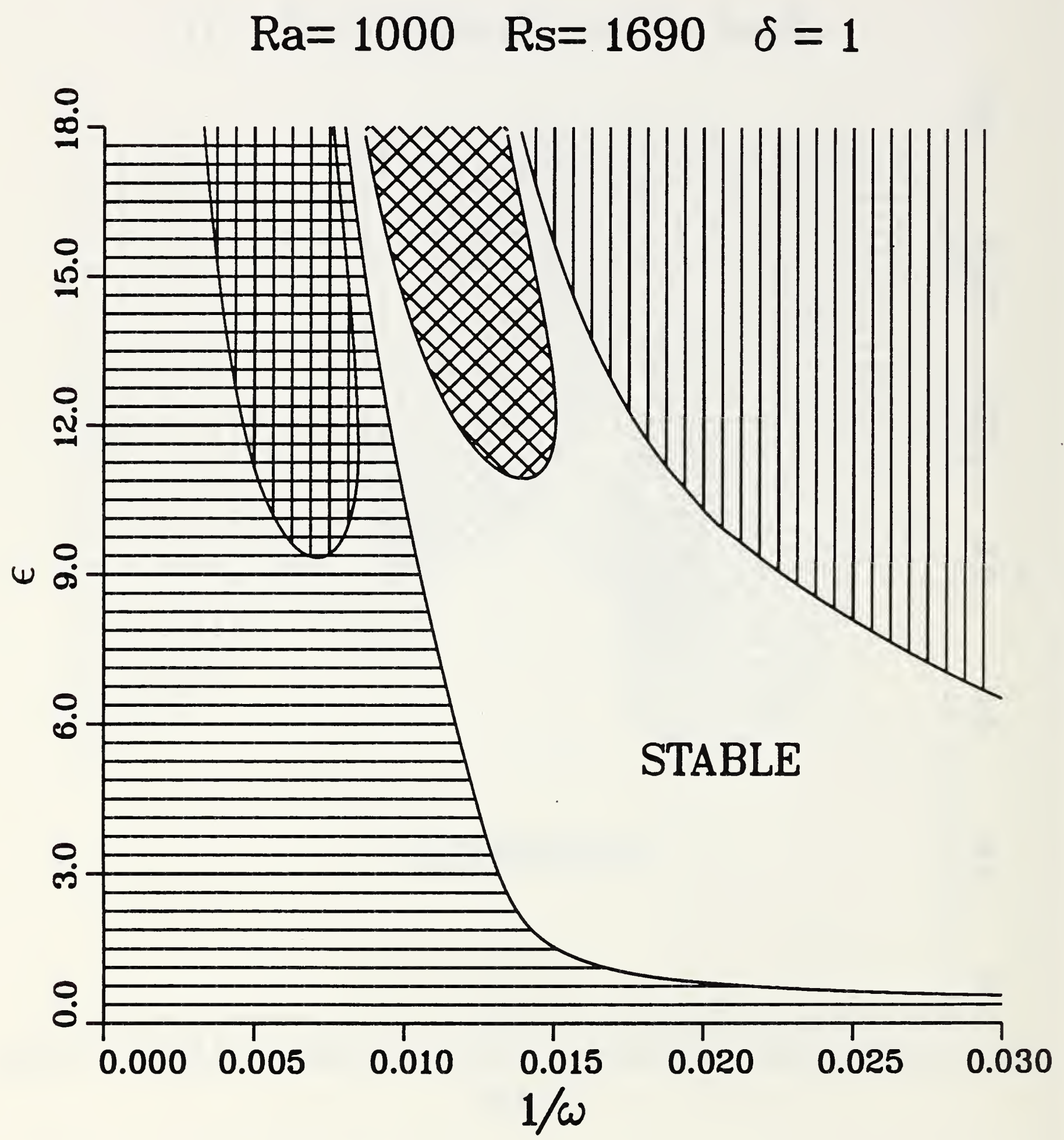

Figure 4 
Modulated Thermosolutal Convection

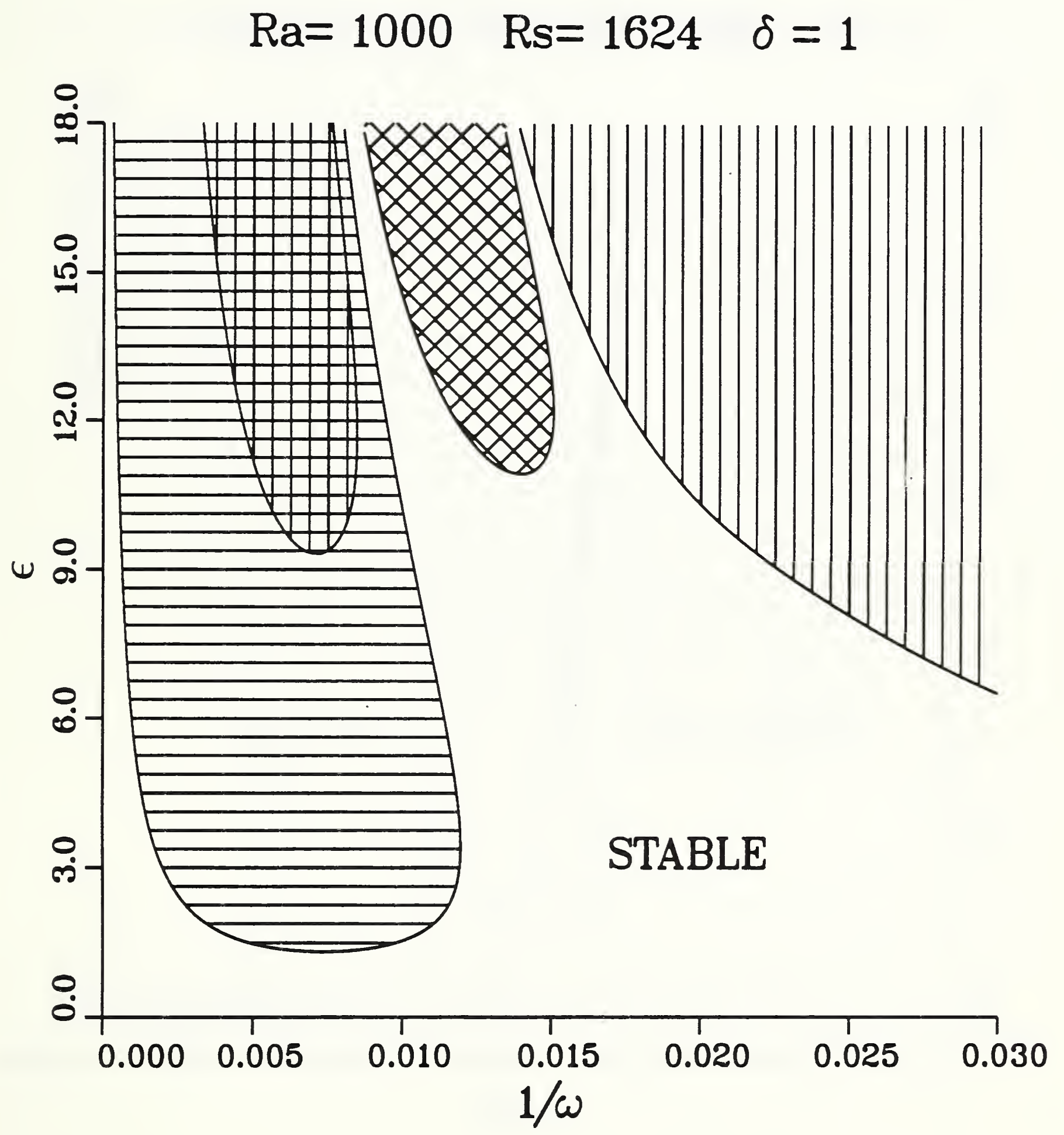


Modulated Thermosolutal Convection

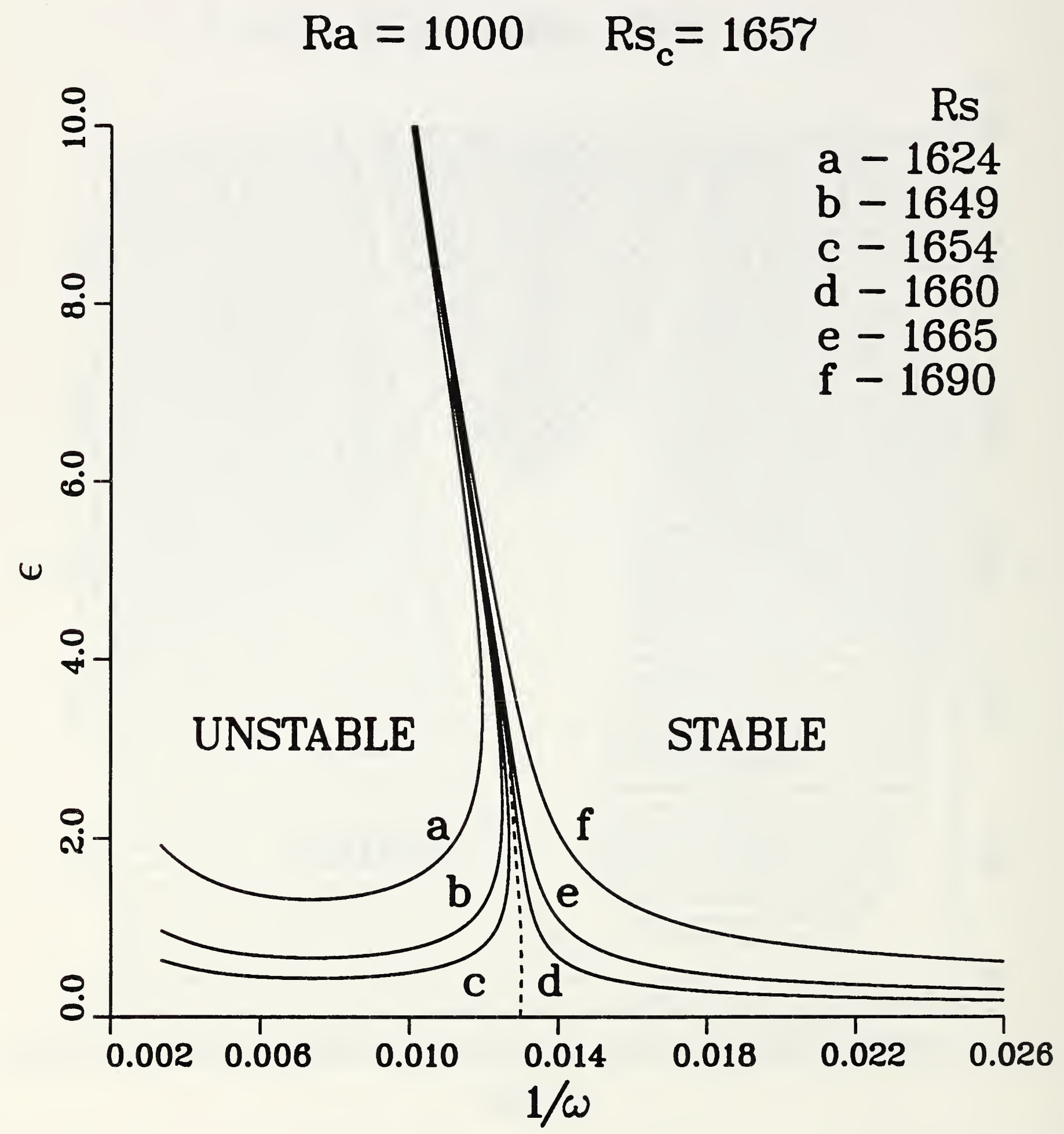


Modulated Thermosolutal Convection

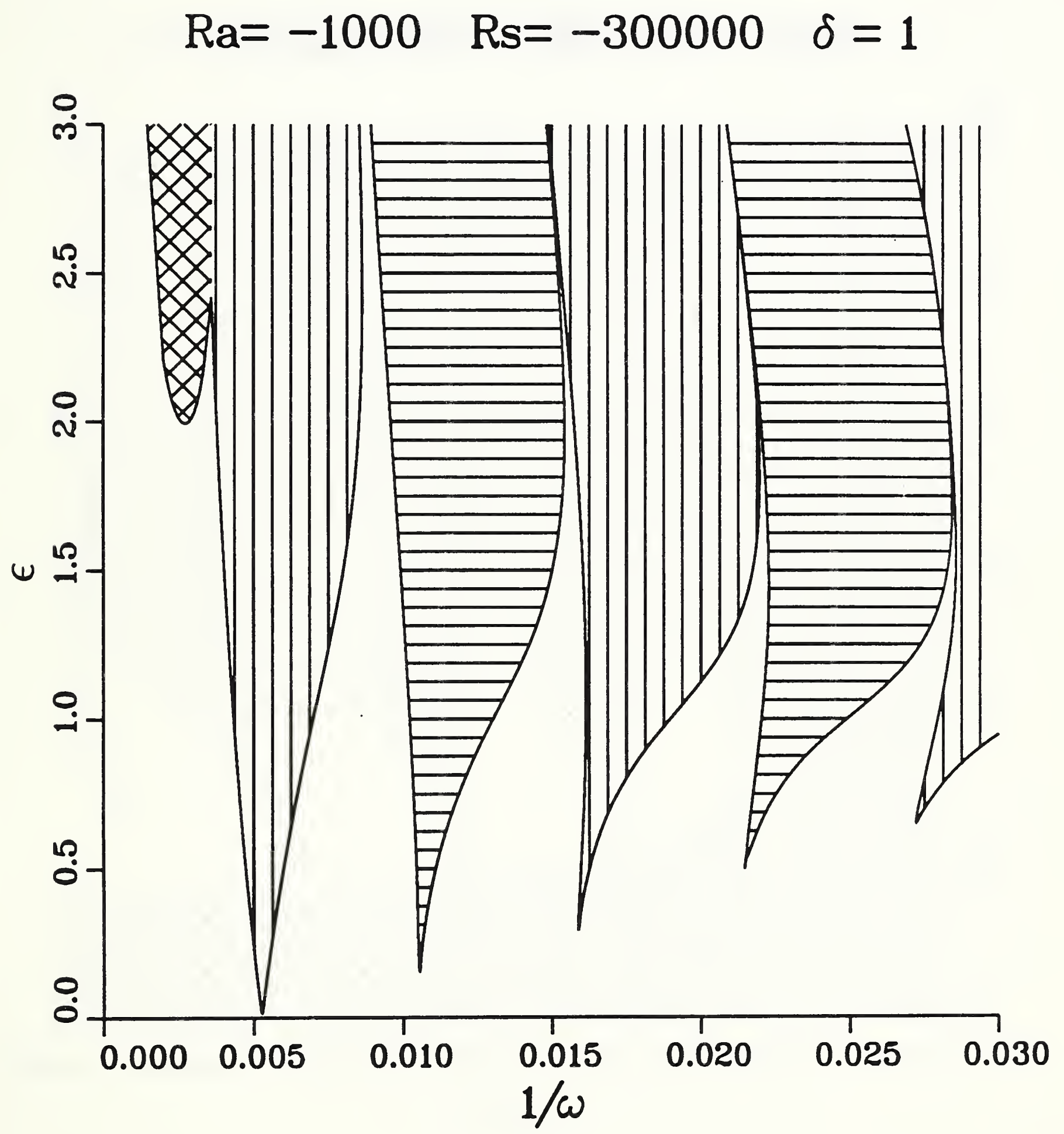


Modulated Thermosolutal Convection

$$
\mathrm{Ra}=-1051 \mathrm{Rs}=-300000 \quad \delta=1
$$

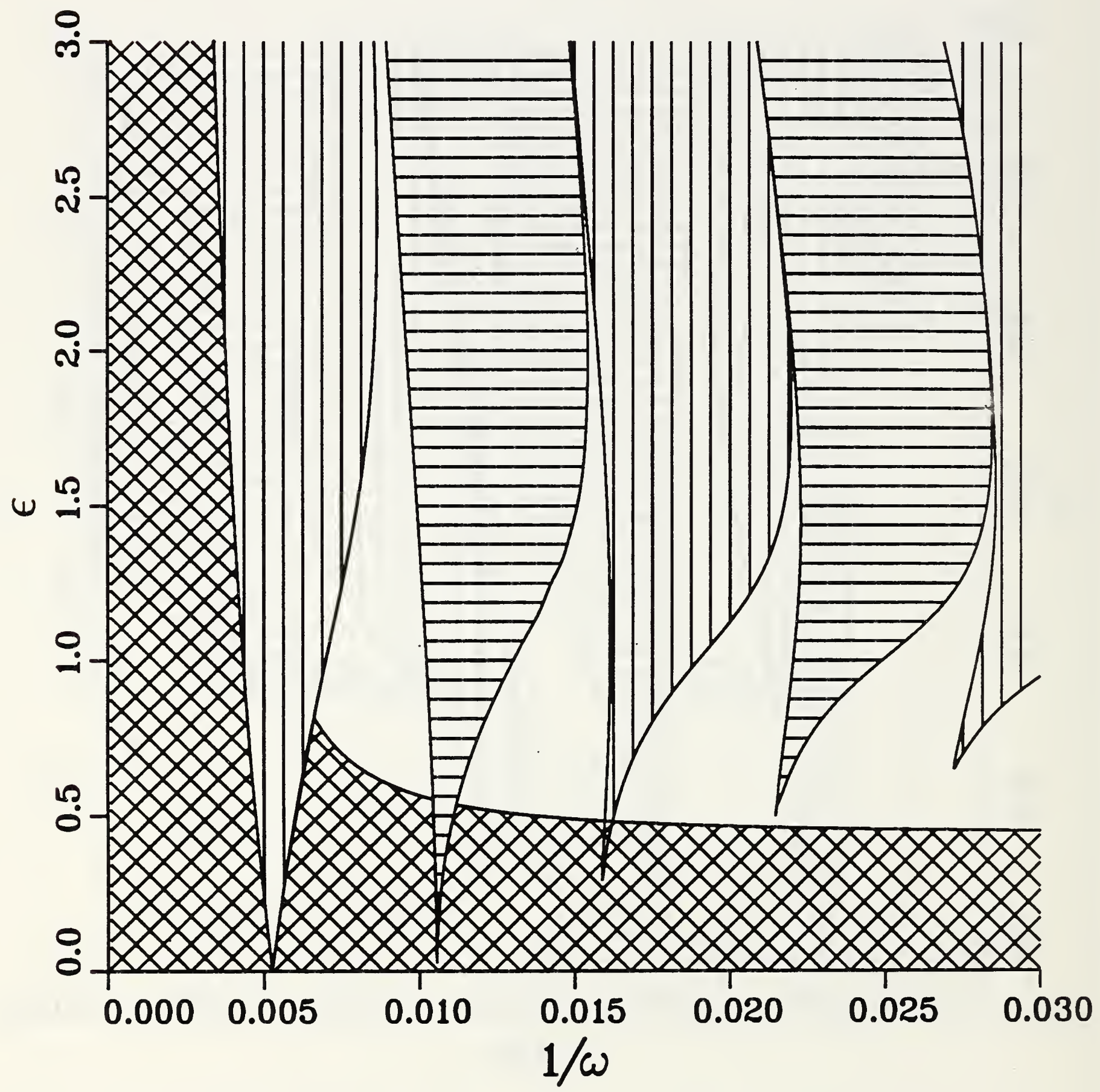


Modulated Solutal Convection

$\mathrm{Ra}=0 \quad \mathrm{Rs}=-300000 \quad \delta=1$

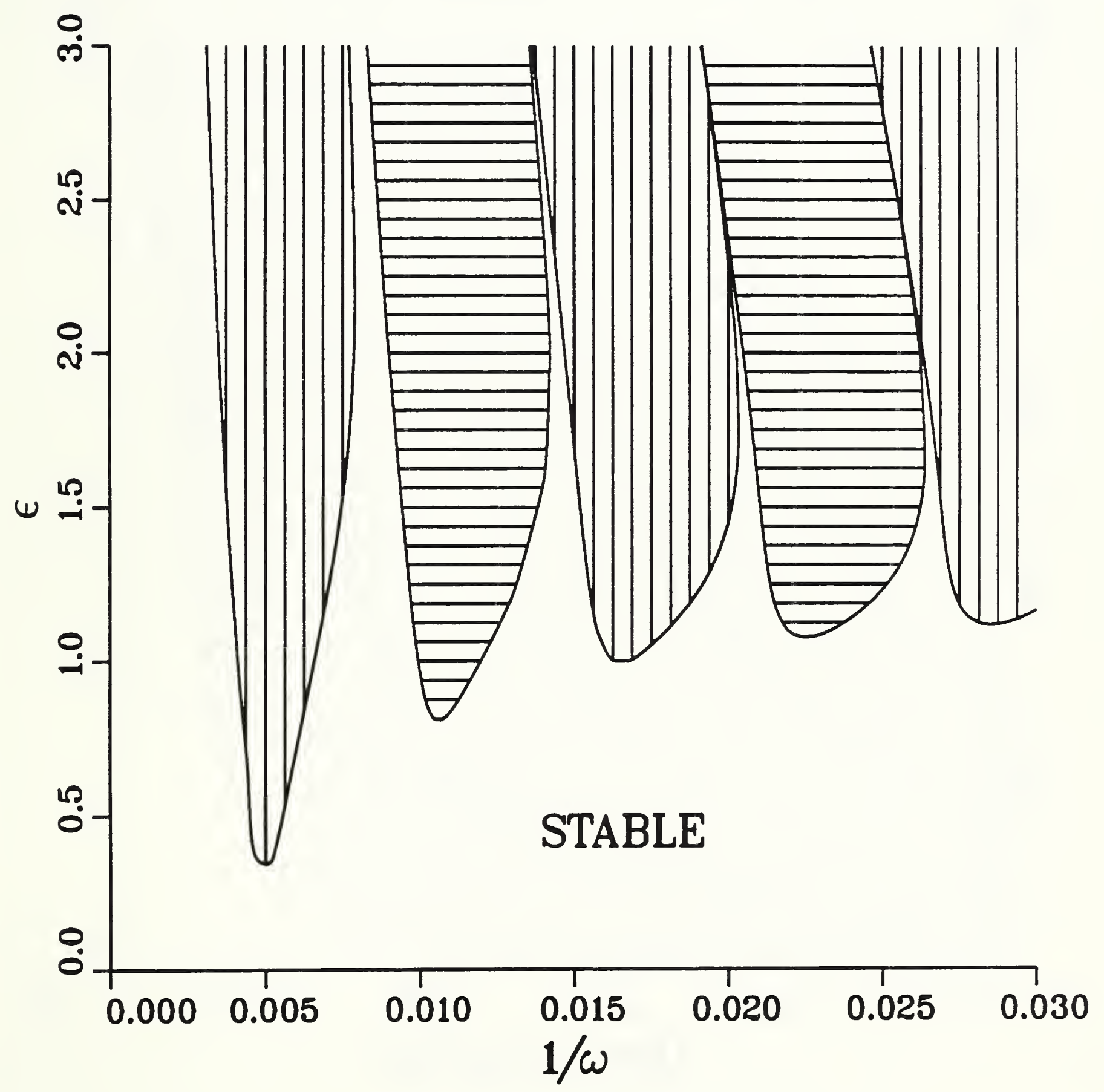




\section{Modulated Thermosolutal Convection}

$$
\mathrm{Rs}=-300000 \quad \delta=1
$$

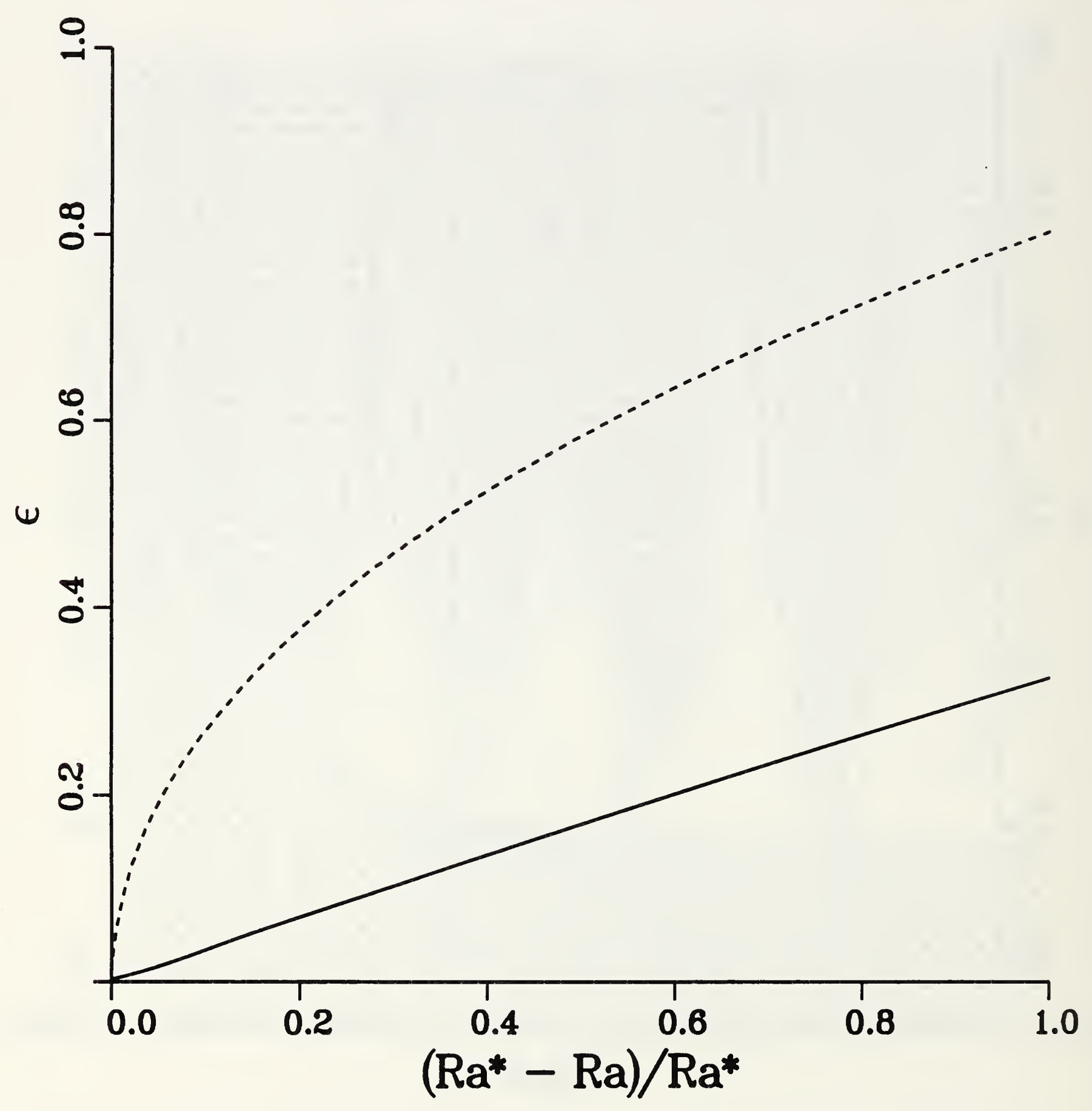

Figure 10 


\section{Modulated Thermosolutal Convection}

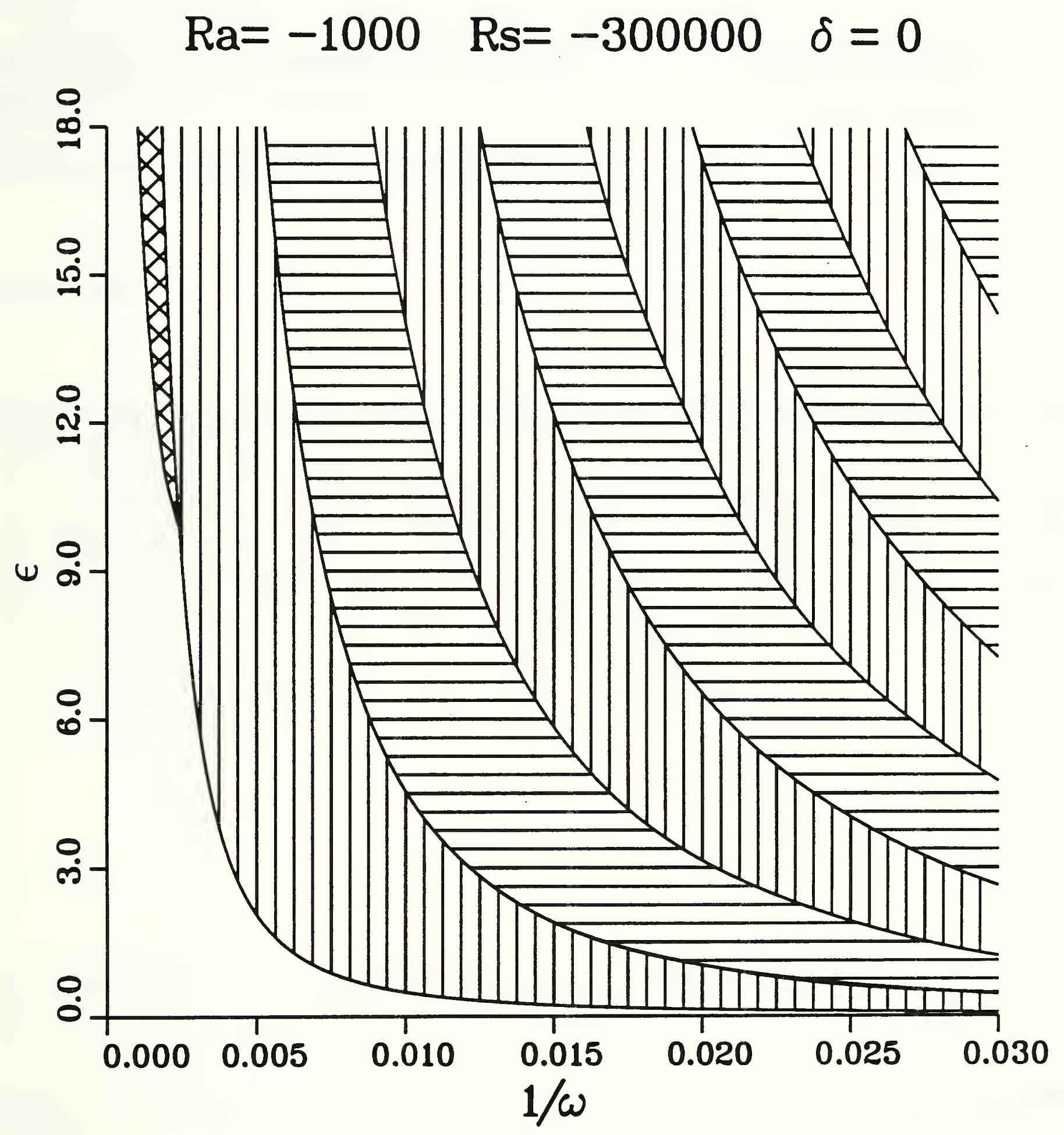





\section{BIBLIOGRAPHIC DATA SHEET}

4. TITLE AND SUBTITLE

The Effect of Gravity Yodulation on Thermosolutal

Convection in an Infinite Layer of Fluid

5. $\operatorname{AUTHOR}(\mathrm{S})$

B. V. Saunders, B. T. Murray, G. B. McFadden, and S. R. Coriell

6. PERFORMINO OROANIZATION (IF JOINT OR OTHER THAN NIST, SEE INSTRUCTIONS)

U.S. DEPARTMENT OF COMMERCE

NATIONAL INSTITUTE OF STANDARDS AND TECHNOLOGY

GAITHERSBURG, MD 20899

7. CONTRACT/GRANT NUMBER

9. SPONSORING ORGANIZATION HAME AND COMPLETE ADDRESS (STREET, CITY, STATE, ZIP)

11. ABSTRACT (A 200-WO RD OR LESS FACTUAL SUMMALYY OF MOST SIGNIFICANT INFORMATION. IF DOCUMENT INCLUDES A SIGNIFICANT BIBLUOGRAPMY OR UTERATURE SURVEY, MENTION IT HERE.)

The effect of time-periodic vertical gravity modulation on the onset of thermosolutal convection in an infinite horizontal laver with stress-free boundaries is investigated using Floquet theory for the linear stability analysis. We consider situations for which the fluid layer is stably stratified in either the fingering or diffusive regimes of doublediffusive convection. Results are presented both with and without steady background acceleration. Modulation may stabilize an unstable base solution or destabilize a stable base solution. In addition to synchronous and subharmonic response to the modulation frequency, instability in the double-diffusive system can occur via a complex conjugate mode. In the diffusive regime, where oscillatory onset occurs in the unmodulated system, regions of resonant instability occur and exhibit strong coupling with the unmodulated oscillatory frequency.

12. KEY WORDS (6 TO 12 ENTRIES; ALPHABETICAL ORDER; CAPITALZE ONLY PROPER NAMES; AND SEPARATE KEY WORDS BY SEMICOLONS) thermosolutal convection; double-diffusive convection; thermohaline convection; buoyant convection; Floquet theory; gravity modulation

FOR OFFICIAL DISTRIBUTION. DO NOT RELEASE TO NATIOHAL TECHMICAL INFORMATION SERVICE (NTIS).

ORDER FROM SUPERINTENDENT OF DOCUMENTS, U.S. GOVERHMENT PRINTING OFFICE, WASHINGTON, DC 20402.

ORDER FROM NATIONAL TECHNICAL IMFORMATION SERVICE (NTIS), SPRINGFIELD, VA 22161.

14. NUMBER OF PRINTED PAGES

$$
41
$$

15. PRICE A03 

